\title{
Probabilistic model identification of uncertainties in computational models for dynamical systems and experimental validation
}

\author{
C. Soize ${ }^{*, a}$, E. Capiez-Lernout ${ }^{a}$, J.-F. Durand ${ }^{a, b}$, \\ C. Fernandez ${ }^{\mathrm{a}, \mathrm{b}}$, L. Gagliardini ${ }^{\mathrm{b}}$ \\ ${ }^{a}$ Université Paris-Est, Laboratoire Modélisation et Simulation Multi Echelle, \\ MSME FRE3160 CNRS, 5 bd Descartes, 77454 Marne la Vallee Cedex 2, France \\ ${ }^{\mathrm{b}}$ PSA Peugeot-Citroen, Route de Gisy, 78943 Velizy-Villacoublay Cedex, France
}

\begin{abstract}
We present a methodology to perform the identification and validation of complex uncertain dynamical systems using experimental data, for which uncertainties are taken into account by using the nonparametric probabilistic approach. Such a probabilistic model of uncertainties allows both model uncertainties and parameter uncertainties to be addressed by using only a small number of unknown identification parameters. Consequently, the optimization problem which has to be solved in order to identify the unknown identification parameters from experiments is feasible. Two formulations are proposed. The first one is the mean-square method for which a usual differentiable objective function and an unusual non-differentiable objective function are proposed. The second one is the maximum likelihood method coupling with a statistical reduction which leads us to a considerable improvement of the method. Three applications with experimental validations are presented in the area of structural vibrations and vibroacoustics.
\end{abstract}

Key words: Identification; Optimization, Uncertain computational model, Structural dynamics, Vibroacoustics, Experimental validation

\footnotetext{
* Corresponding author. Tel.: 33160957661 ; fax: 33160957799

Email address: christian.soize@univ-paris-est.fr (C. Soize ).
} 


\section{Introduction}

Uncertainty modeling and quantification in computational mechanics have received particularly attention in recent years. Statistical and probabilistic procedures have provided a sound framework for a rational basis for processing uncertainties. In this context the aspects of model validation and verification respectively are of interest.

The updating (or the identification) of a computational model and the design optimization with a computational model require to solve optimization problems of the same nature. When uncertainties are taken into account in such a computational model, then the corresponding optimization problems are known as robust updating (or robust identification) and robust design optimization. For instance, the updating (or the identification) of a computational model using experimental data is currently a challenge of interest in structural dynamics $[4,15,21,29,30,35,36,38,43,50]$ and the design optimization with a computational model is a subject of importance in structural acoustics $[5,17,23,40]$. Nowadays it is well understood that the effects of uncertainties have to be taken into account to update (or to indentify) a computational model and to optimize a design with a computational model. For instance, robust updating (or robust identification) has recently been proposed in the context of structural dynamics $[34,41,42,45]$. Similarly, robust design optimization has been proposed in different areas $[16,33,37,46,47,49,51,65,67]$. It should be noted that such robust updating (or robust identification) and robust design optimization are developed in the context of parameter uncertainties but not in the context of both parameter uncertainties and model uncertainties. Recently, robust design optimization has been analyzed with model uncertainties [6].

The context of this paper is the validation of probability models of uncertainties in the computational models used for the analysis of complex dynamical systems. These computational models are constructed by developing a mathematical-physical model of the designed dynamical system conceived by the designers and analysts. Such a computational model exhibits an input, an output and a vector-valued parameter. The real dynamical system is a manufactured version of the system realized from the designed dynamical system. Consequently, the real dynamical system is a man made physical system which is never exactly known due to the variability induced by the manufacturing process and by small differences in the configurations. The computational model is used either to predict the response of the real dynamical system in including its variabilities or to optimize the design. The mathematical physical modeling process of the designed dynamical system introduces two fundamental types of uncertainties: the parameter uncertainties and the model uncertainties. It is important to note that the model uncertainties are not 
due to external noises or cannot be represented by equivalent external noises. The manufacturing process induces variabilities in the real dynamical system. In general, no experimental measurements are available for the responses of such complex dynamical systems. Consequently, the parameter uncertainties and the model uncertainties have to be taken into account in the computational model in order to improve the predictability and the robustness of the predictions. We then need to model the uncertainties in the dynamical system. It is known that several approaches can be used to take into account the uncertainties in the computational models of complex dynamical systems for the low and medium frequencies (method of intervals, fuzzy sets, probabilistic approach, etc). The most efficient and the most powerful mathematical tool adapted to model the uncertainties is the probabilistic approach as soon as the probability theory can be used. The parametric probabilistic approach which includes the stochastic finite element method is the most efficient method to address the parameter uncertainties in the predictive models. Such an approach consists in modeling the vector-valued parameter by a vector-valued random variable for which the probability distribution must be constructed using the information theory [56] and the available information (for instance, consisting of given algebraic properties and of a given mean value). If a lot of experimental data are available, then the Bayesian approach can be used to update such a probability distribution (see for instance [64] and [66]). However, the parametric probabilistic approach cannot address the model uncertainties as it is demonstrated, for example, in [61] and [62]. In addition, the use of the parametric probabilistic approach of the parameter uncertainties for the analysis of a complex dynamical system generally requires the introduction of a very large number of random variables. This is due to the fact that the dynamical responses can be very sensitive to many parameters relative to the boundary conditions or to the geometry (such as the thicknesses of plates or as the curvatures of panels), etc. Typically, several hundred thousands random variables can be necessary to take into account parameter uncertainties in a complex dynamical system. It should be noted that the construction of the probabilistic model for a large number of random parameters is not so easy to carry out. In addition, for such a complex dynamical system there are generally no available measurements. If very little measurements are available, then the identification of a large number of probability distributions using experimental data (using the Bayesian approach, the maximum likelihood method or another approach leading an optimization problem to be solved) can become unrealistic. In this context for which no measurements are available or for which very little measurements are available, it can be concluded that very little can be learnt from the experimental data. The idea is then to develop an approach for which the uncertain computational model can mainly learn from the available information (such as algebraic properties and given mean values) but not from experimental data. The nonparametric probabilistic approach [57-63] recently proposed and based on the use of the random matrix theory is a way to address both model uncertainties and parameter uncertain- 
ties without using experimental data. This nonparametric approach proposed introduces a very small number of parameters (typically 7 parameters for a structural-acoustic system) which allows the level of uncertainties to be controlled. In the particular case for which very little measurements are available, the identification of these parameters using experimental data is realistic and can be performed by solving adapted mathematical statistical tools such as the mean-square method or the maximum likelihood method.

The objective of this paper is to present formulations for experimental validation of complex uncertain dynamical systems for which uncertainties are taken into account by using the nonparametric probabilistic approach and for which very little measurements are available. Consequently, the optimization problem which has to be solved in order to identify the unknown identification parameters from experiments is feasible for very large computational dynamical model. It should be noted that when the parameters of this nonparametric probabilistic model have been identified for one complex dynamical system belonging to a large class of dynamical systems representing many different configurations, this probabilistic model can be reused to analyze or to optimize another design belonging to this large class without needing experimental data. This important property is due to the fact that the nonparametric probabilistric approach consists in constructing a probabilistic model of the operators of the problem using intrinsic available information relative to the operators of the dynamical system such as the mass, damping, stiffness, structural-acoustic coupling operators. Three formulations are proposed. The first one is based on the usal mean-square method with a differentiable objective function. The second one introduces a new unusual non-differentiable objective function which is particularly well adapted to the responses of dynamical systems formulated in the frequency domain. The last one is a new methodology which is very efficient for dynamical systems formulated in the frequency domain and which is based on the use of the maximum likelihood method coupled with a statistical reduction in the frequency domain and which leads us to a considerable improvement of the method. Three applications with experimental validations will be presented in the area of structural vibrations and vibroacoustics to illustrate the different methods proposed.

\section{Reduced mean computational model}

In this paper, we consider a linear elastodynamic problem (structural dynamics) or a linear elastoacoustic problem (structural acoustics of a structure coupled with a bounded internal acoustic cavity). It is assumed that the computational model is constructed using the finite element method and is formulated in the frequency domain $\omega$ (angular frequency). A reduced mean computational model is constructed with the usual method of projection on 
subspaces spanned by a set of elastic modes of the structure in vacuo and/or a set of acoustic modes of the acoustic cavity with rigid walls. This means that the domain of validity of such a reduced mean computational model is the lowand medium-frequency ranges and the frequency band of analysis is denoted by $B=\left[\omega_{\min }, \omega_{\max }\right]$ with $0 \leq \omega_{\min }<\omega_{\max }$. The reduced mean computational model is then written as

$$
\begin{aligned}
& \underline{\mathbf{v}}(\mathbf{r}, \omega)=\left[\Phi\left(\mathbf{r}_{0}\right)\right] \underline{\mathbf{q}}(\mathbf{r}, \omega), \\
& {[\underline{a}(\mathbf{r}, \omega)] \underline{\mathbf{q}}(\mathbf{r}, \omega)=\left[\Phi\left(\mathbf{r}_{0}\right)\right]^{T} \underline{\mathbf{f}}(\mathbf{r}, \omega),} \\
& \underline{\mathbf{w}}(\mathbf{r}, \omega)=\underline{\mathbf{h}}(\omega, \underline{\mathbf{v}}(\mathbf{r}, \omega)) .
\end{aligned}
$$

In Eqs. (1) to (3), the parameter $\mathbf{r}$ belongs to an admissible set $\mathcal{R}$ which is a subset of $\mathbb{R}^{n_{r}}$ (with $n_{r} \geq 1$ ). Parameter $\mathbf{r}$ represents either an updating parameter or a design parameter which has to be identified and which will be called the identification parameter of the mean computational model. The parameter $\mathbf{r}_{0}$ is a given nominal value of $\mathbf{r}$. For all $\mathbf{r}$ fixed in $\mathcal{R}$ and for all $\omega$ fixed in $B$ :

(i) $\mathbf{v}(\mathbf{r}, \omega)$ is the complex vector in $\mathbb{C}^{n_{v}}$ of the degrees of freedom (structural displacements and/or acoustic pressures) of the mean computational model with $n_{v}>1$.

(ii) $\mathbf{f}(\mathbf{r}, \omega)$ is a complex vector in $\mathbb{C}^{n_{v}}$ which represents the loads due to forces applied to the structure and/or the internal acoustic sources.

(iii) $\underline{\mathbf{w}}(\mathbf{r}, \omega)$ is a real vector in $\mathbb{R}^{n}$ with $n \geq 1$ and represents the real-valued observation of the mean computational model. For instance, we will have $\underline{w}_{j}(\mathbf{r}, \omega)=20 \log _{10}\left(\omega^{2}\left|\underline{v}_{k_{j}}(\mathbf{r}, \omega)\right|\right)$ in which $v_{k_{j}}(\mathbf{r}, \omega)$ will be a structural displacement or $\underline{w}_{\ell}(\mathbf{r}, \omega)=20 \log _{10}\left(\left|\underline{p}_{k_{\ell}}(\mathbf{r}, \omega)\right|\right)$ in which $\underline{p}_{k_{\ell}}(\mathbf{r}, \omega)$ will be an acoustic pressure. Such equations define the mapping $\mathbf{v} \mapsto \mathbf{w}=\underline{\mathbf{h}}(\omega, \mathbf{v})$ from $\mathbb{C}^{n_{v}}$ in $\mathbb{R}^{n}$.

(iv) $\left[\Phi\left(\mathbf{r}_{0}\right)\right]$ is a $\left(n_{v} \times N\right)$ real matrix whose columns are made up of elastic modes of the structure in vacuo and/or acoustic modes of the acoustic cavity with rigid wall, computed for the nominal value $\mathbf{r}_{0}$ of the updating parameter r. The matrix $\left[\Phi\left(\mathbf{r}_{0}\right)\right]^{T}$ is the transpose of $\left[\Phi\left(\mathbf{r}_{0}\right)\right]$.

(v) $\underline{\mathbf{q}}(\mathbf{r}, \omega)$ is the complex vector in $\mathbb{C}^{N}$ of the generalized coordinates of the reduced mean computational model.

(vi) $[\underline{a}(\mathbf{r}, \omega)]$ is the $(N \times N)$ complex matrix representing the generalized stiffness matrix. 
It is assumed that for all $\mathbf{r}$ in $\mathcal{R}$ and for all $\omega$ in $B$, dimension $N$ of the reduced computational model is sufficiently large (but $N \ll n_{v}$ ) to reach a reasonable convergence.

\section{Construction of the stochastic computational model to take into account uncertainties}

It is assumed that there are uncertainties in the mean computational model. There are two main types of uncertainties, parameter uncertainties and model uncertainties.

Usually, parameter uncertainties are taken into account by using a parametric probabilistic approach allowing uncertain parameters of the computational model to be modeled by random variables, random fields, etc. Such a parametric probabilistic approach is the most efficient method to address parameter uncertainties in a computational model (see for instance, [52-54]). In particular, the stochastic finite element method is a powerful tool to analyze propagation of parameter uncertainties through the computational model associated with a boundary value problem (see for instance, [24,25]).

The mathematical-mechanical modeling process of the designed system used to construct the computational model introduces model uncertainties which cannot be addressed by the parametric probabilistic approach (see [61]). In this paper, it is assumed that parameter uncertainties and model uncertainties exist in the computational model. Consequently, we propose to use the nonparametric probabilistic approach of both parameter uncertainties and model uncertainties recently introduced (see [57] to [63]), constructed by using the maximum entropy principle $[31,56]$, and for which experimental validations can be found in $[8-10,18,19]$. The maximum entropy principle was introduced by Shannon [56] in the construction of the information theory. This principle consists in constructing the probability density function which maximizes the uncertainties under the constraints defined by the available information. Applying the nonparametric probabilistic approach to Eqs. (1) to (3) yields the following stochastic reduced model such that, for all $\omega$ in $B$ and for all $\mathbf{r}$ in $\mathcal{R}$,

$$
\begin{aligned}
& \mathbf{V}(\mathbf{r}, \boldsymbol{\delta}, \omega)=\left[\Phi\left(\mathbf{r}_{0}\right)\right] \mathbf{Q}(\mathbf{r}, \boldsymbol{\delta}, \omega), \\
& {[\mathbf{A}(\mathbf{r}, \boldsymbol{\delta}, \omega)] \mathbf{Q}(\mathbf{r}, \boldsymbol{\delta}, \omega)=\left[\Phi\left(\mathbf{r}_{0}\right)\right]^{T} \underline{\mathbf{f}}(\mathbf{r}, \omega),} \\
& \mathbf{W}(\mathbf{r}, \boldsymbol{\delta}, \omega)=\underline{\mathbf{h}}(\omega, \mathbf{v}(\mathbf{r}, \boldsymbol{\delta}, \omega)),
\end{aligned}
$$

in which all the random quantities are defined on a probability space $(\Theta, \mathcal{T}, \mathcal{P})$. The random matrix $[\mathbf{A}(\mathbf{r}, \boldsymbol{\delta}, \omega)]$ is a $(N \times N)$ complex random matrix for which 
the probability distribution is known and which is such that

$$
E\{[\mathbf{A}(\mathbf{r}, \delta, \omega)]\}=[\underline{a}(\mathbf{r}, \omega)],
$$

where $E$ is the mathematical expectation. This random matrix depends on the random matrix germs modeling uncertainties and for which their probability distributions depend on a vector parameter $\delta$ belonging to a subset $\Delta$ of $\mathbb{R}^{n_{\delta}}$. The probability model of the random matrix germs and the corresponding generator of independent realizations can be found in references [57] to [63] and in particular, in reference [62]. Some additional explanations are given in Section 6 devoted to the applications. Parameter $\delta$ allows the dispersion induced by uncertainties to be controlled [62]. We will give the construction of such a probability model in the applications presented in Section 6. The probability model used is such that, for all $\mathbf{r}$ in $\mathcal{R}$, for all $\delta$ in $\Delta$ and for all $\omega$ in $B$, the random equation (5) has a unique second-order random solution, i.e. $E\left\{\|\mathbf{Q}(\mathbf{r}, \boldsymbol{\delta}, \omega)\|_{N}^{2}\right\}<+\infty$ in which $\|\mathbf{z}\|_{N}^{2}=\left|z_{1}\right|^{2}+\ldots+\left|z_{N}\right|^{2}$.

\section{Definition of the identification parameters}

In this paper, the identification problem which has to be solved is

(i) either the identification of parameter $\delta \in \Delta \subset \mathbb{R}^{n_{\delta}}$ of the probability model of uncertainties for a fixed value of parameter $\mathbf{r} \in \mathcal{R}$ of the mean computational model using experimental data. In this case vector-valued random observation $\mathbf{W}(\mathbf{r}, \delta, \omega)$ is rewritten as $\mathbf{W}(\mathbf{s}, \omega)$ in which dependence with $\mathbf{r}$ is removed and where $\mathbf{s}$ belongs to the subset $\mathcal{S}$ of $\mathbb{R}^{n_{s}}$ with $\mathbf{s}=\delta, \mathcal{S}=\Delta$ and $n_{s}=n_{\delta}$.

(ii) or the identification of parameter $\mathbf{r} \in \mathcal{R} \subset \mathbb{R}^{n_{r}}$ of the mean computational model and of parameter $\delta \in \Delta \subset \mathbb{R}^{n_{\delta}}$ of the probability model of uncertainties using experimental data. In this case, the vector-valued random observation $\mathbf{W}(\mathbf{r}, \boldsymbol{\delta}, \omega)$ is also rewritten as $\mathbf{W}(\mathbf{s}, \omega)$ in which $\mathbf{s}=(\mathbf{r}, \boldsymbol{\delta}) \in \mathcal{S}=\mathcal{R} \times \Delta \subset \mathbb{R}^{n_{s}}=$ $\mathbb{R}^{n_{r}} \times \mathbb{R}^{n_{\delta}}$ with $n_{s}=n_{r}+n_{\delta}$.

Consequently, we will define $\mathbf{s} \in \mathcal{S} \subset \mathbb{R}^{n_{s}}$ as the identification parameter (the parameter which has to be identified using experimental measurements of the vector-valued observation). 


\section{Identification method of the identification parameter using ex- perimental observations of the system}

\subsection{Setting the problem}

Let $\omega \mapsto \mathbf{W}(\mathbf{s}, \omega)$ be the second-order stochastic process defined on $(\Theta, \mathcal{T}, \mathcal{P})$ indexed by $B$, with values in $\mathbb{R}^{n}$ and depending on the identification parameter $\mathbf{s} \in \mathcal{S} \subset \mathbb{R}^{n_{s}}$. Let $\left\{\omega_{1}, \ldots, \omega_{m}\right\} \subset B$ be a sampling of frequency band $B$. Let $\mu_{1}, \ldots, \mu_{m}$ be the integration weights associated with $\left\{\omega_{1}, \ldots, \omega_{m}\right\}$ such that

$$
\int_{B} d \omega=\sum_{k=1}^{m} \mu_{k} \quad, \quad \int_{B} f(\omega) d \omega \simeq \sum_{k=1}^{m} \mu_{k} f\left(\omega_{k}\right) .
$$

For all $\mathbf{s}$ fixed in $\mathcal{S}$, let $\mathbf{w}\left(\mathbf{s}, \omega_{1}\right), \ldots, \mathbf{W}\left(\mathbf{s}, \omega_{m}\right)$ be the finite family of the $\mathbb{R}^{n_{-}}$ valued random variables associated with the uncountable family $\{\mathbf{W}(\mathbf{s}, \omega), \omega \in$ $B\}$. In general, random variables $\mathbf{W}\left(\mathbf{s}, \omega_{1}\right), \ldots, \mathbf{W}\left(\mathbf{s}, \omega_{m}\right)$ are statistically dependent. Let $P_{\mathbf{w}\left(\mathbf{s}, \omega_{1}\right), \ldots, \mathbf{w}\left(\mathbf{s}, \omega_{m}\right)}\left(d \mathbf{w}^{1}, \ldots, d \mathbf{w}^{m}, \mathbf{s}\right)$ be the joint probability distribution on $\mathbb{R}^{n} \times \ldots \times \mathbb{R}^{n}(m$ times $) \simeq \mathbb{R}^{n m}$ of random variables $\mathbf{W}\left(\mathbf{s}, \omega_{1}\right), \ldots, \mathbf{W}\left(\mathbf{s}, \omega_{m}\right)$ depending on parameter $\mathbf{s}$ in which $d \mathbf{w}^{k}=d w_{1}^{k} \ldots d w_{n}^{k}$ is the Lebesgue measure on $\mathbb{R}^{n}$ for all $k=1, \ldots, m$. For all $\mathbf{s}$ fixed in $\mathcal{S}$, this joint probability distribution is assumed to be written as

$$
P_{\mathbf{w}\left(\mathbf{s}, \omega_{1}\right), \ldots, \mathbf{w}\left(\mathbf{s}, \omega_{m}\right)}\left(d \mathbf{w}^{1}, \ldots, d \mathbf{w}^{m}, \mathbf{s}\right)=p\left(\mathbf{w}^{1}, \ldots, \mathbf{w}^{m}, \mathbf{s}\right) d \mathbf{w}^{1} \ldots d \mathbf{w}^{m}
$$

in which $p\left(\mathbf{w}^{1}, \ldots, \mathbf{w}^{m}, \mathbf{s}\right)$ is the probability density function on $\mathbb{R}^{n} \times \ldots \times \mathbb{R}^{n}$ $(m$ times $) \simeq \mathbb{R}^{n m}$ with respect to $d \mathbf{w}^{1} \ldots d \mathbf{w}^{m}$ and where $\mathbf{w}^{k}=\left(w_{1}^{k}, \ldots, w_{n}^{k}\right)$. For all $\mathbf{s}$ fixed in $\mathcal{S}$ and for all vectors $\mathbf{w}^{1}, \ldots, \mathbf{w}^{m}$ given in $\mathbb{R}^{n}$, the estimation of $p\left(\mathbf{w}^{1}, \ldots, \mathbf{w}^{m}, \mathbf{s}\right)$ is performed by using the stochastic computational model and the Monte Carlo method as stochastic solver with $\nu$ independent realizations $\left\{\mathbf{W}\left(\mathbf{s}, \omega_{1} ; \theta_{1}\right), \ldots, \mathbf{W}\left(\mathbf{s}, \omega_{m} ; \theta_{1}\right)\right\}, \ldots,\left\{\mathbf{W}\left(\mathbf{s}, \omega_{1} ; \theta_{\nu}\right), \ldots, \mathbf{W}\left(\mathbf{s}, \omega_{m} ; \theta_{\nu}\right)\right\}$ of the $\mathbb{R}^{n m_{-}}$ valued random observations $\left\{\mathbf{W}\left(\mathbf{s}, \omega_{1}\right), \ldots, \mathbf{W}\left(\mathbf{s}, \omega_{m}\right)\right\}$ with $\theta_{1}, \ldots, \theta_{\nu}$ in $\Theta$. This means that:

(1) any independent realization $\left[\mathbf{A}\left(\mathbf{s}, \omega_{k} ; \theta_{\ell}\right)\right]$ of the random matrix $\left[\mathbf{A}\left(\mathbf{s}, \omega_{k}\right)\right]$ is constructed using the probability model introduced in Section 3. This probability model is completely defined and there exists an associated generator of independent realizations (see the given references and also the applications presented in Section 6).

(2) the corresponding realization $\mathbf{W}\left(\mathbf{s}, \omega_{k} ; \theta_{\ell}\right)$ is calculated using the computational stochastic model defined by Eqs.(4) to (6), that is to say, is given by $\mathbf{W}\left(\mathbf{s}, \omega_{k} ; \theta_{\ell}\right)=\underline{\mathbf{h}}\left(\omega_{k}, \mathbf{V}\left(\mathbf{s}, \omega_{k} ; \theta_{\ell}\right)\right)$ with $\mathbf{v}\left(\mathbf{s}, \omega_{k} ; \theta_{\ell}\right)=\left[\Phi\left(\mathbf{r}_{0}\right)\right] \mathbf{Q}\left(\mathbf{s}, \omega_{k} ; \theta_{\ell}\right)$ in which 
the deterministic vector $\mathbf{Q}\left(\mathbf{s}, \omega_{k} ; \theta_{\ell}\right)$ is the solution of the deterministic matrix equation $\left[\mathbf{A}\left(\mathbf{s}, \omega_{k} ; \theta_{\ell}\right)\right] \mathbf{Q}\left(\mathbf{s}, \omega_{k} ; \theta_{\ell}\right)=\left[\Phi\left(\mathbf{r}_{0}\right]^{T} \mathbf{f}\left(\mathbf{r}, \omega_{k}\right)\right.$.

(3) finally, the probability density function $p\left(\mathbf{w}^{1}, \ldots, \mathbf{w}^{m}, \mathbf{s}\right)$ or any moments are estimated using the appropriate estimators given by the mathematical statistics.

Concerning the experimental observations, we consider $\nu_{\exp }$ manufactured systems $\eta_{1}, \ldots, \eta_{\nu_{\exp }}$ with $\nu_{\exp } \geq 1$. In general, $\nu_{\text {exp }}$ is small (one unit or a few units). These $\nu_{\text {exp }}$ manufactured systems are considered as $\nu_{\text {exp }}$ independent realizations of a random manufactured system. We then denote by $\left\{\mathbf{W}^{\exp }(\omega), \omega \in B\right\}$ the random observation defined on a probability space $\left(\Theta_{\text {exp }}, \mathcal{T}_{\text {exp }}, \mathcal{P}_{\text {exp }}\right)$ of the random manufactured system corresponding to the random observation $\{\mathbf{W}(\omega)$, $\omega \in B\}$ of the stochastic computational model. By construction, we then have $\eta_{1}, \ldots, \eta_{\nu_{\text {exp }}}$ in $\Theta_{\text {exp }}$. The experimental observation of the manufactured system $\eta_{\ell}$ corresponds to the realization $\left\{\mathbf{W}^{\exp }\left(\omega, \eta_{\ell}\right), \omega \in B\right\}$ of the random experimental observation $\left\{\mathbf{W}^{\exp }(\omega), \omega \in B\right\}$ of the random manufactured system. In practice, the sampling $\left\{\omega_{1}, \ldots, \omega_{m}\right\}$ of $B$ is used and the experimental observation of the manufactured system $\eta_{\ell}$ is made up of the finite family of the $\mathbb{R}^{n}$-valued experimental observations $\left\{\mathbf{W}^{\exp }\left(\omega_{1} ; \eta_{\ell}\right), \ldots, \mathbf{W}^{\exp }\left(\omega_{m} ; \eta_{\ell}\right)\right\}$. Consequently, for $\mathbf{s}$ fixed in $\mathcal{S}$, the experimental observations corresponding to the random observation $\left\{\mathbf{W}\left(\mathbf{s}, \omega_{1}\right), \ldots, \mathbf{W}\left(\mathbf{s}, \omega_{m}\right)\right\}$ of the stochastic computational model are made up of

$\left\{\mathbf{W}^{\exp }\left(\omega_{1} ; \eta_{1}\right), \ldots, \mathbf{W}^{\exp }\left(\omega_{m} ; \eta_{1}\right)\right\}, \ldots,\left\{\mathbf{W}^{\exp }\left(\omega_{1} ; \eta_{\nu_{\exp }}\right), \ldots, \mathbf{W}^{\exp }\left(\omega_{m} ; \eta_{\nu_{\exp }}\right)\right\}$

which are $\nu_{\text {exp }}$ independent realizations of the random experimental observation $\left\{\mathbf{W}^{\exp }\left(\omega_{1}\right), \ldots, \mathbf{W}^{\exp }\left(\omega_{m}\right)\right\}$. The problem to be solved is then the identification of the optimal value $\mathbf{s}^{\text {opt }}$ of the identification parameter $\mathbf{s}$ of the stochastic computational model using the experimental values defined by Eq. (10). Below, we propose to use two main methods: (1) the mean-square method for differentiable and not differentiable objective functions and (2) the maximum likelihood method.

\subsection{Mean-square method}

This section deals with the usual mean-square method [64], [66]. Two cases will be considered: the case of a differentiable objective function and the case of a non-differentiable objective function. These two cases will be used in the applications presented in Section 6 .

We begin by introducing an inner product which is adapted to the sampled frequency random observations $\left\{\mathbf{W}\left(\mathbf{s}, \omega_{1}\right), \ldots, \mathbf{W}\left(\mathbf{s}, \omega_{m}\right)\right\}$ of the stochastic com- 
putational model and to its corresponding sampled random experimental observation $\left\{\mathbf{W}^{\exp }\left(\omega_{1}\right), \ldots, \mathbf{W}^{\exp }\left(\omega_{m}\right)\right\}$.

Let $\boldsymbol{X}=\left(\mathbf{X}^{1}, \ldots, \mathbf{X}^{m}\right)$ and $\mathbb{Y}=\left(\mathbf{Y}^{1}, \ldots, \mathbf{Y}^{m}\right)$ be two random variables with values in $\mathbb{R}^{n} \times \ldots \times \mathbb{R}^{n}$ ( $m$ times $) \simeq \mathbb{R}^{n m}$ in which, for all $j=1, \ldots, m, \mathbf{X}^{j}$ and $\mathbf{Y}^{j}$ are random variables with values in $\mathbb{R}^{n}$. This algebraic structure of random vectors $\mathcal{X}$ and $\boldsymbol{\Psi}$ is adapted to the frequency sampled random observations over frequency band $B$. We then introduce the inner product $\ll \mathbb{x}, \mathbb{Y} \gg$ of $\mathbb{X}$ and $\checkmark$ such that

$$
\left.\left.\ll \mathfrak{X}, \mathbb{Y} \gg=E\{<\mathfrak{X}, \mathbb{Y}\rangle_{B}\right\} \quad, \quad<\mathfrak{X}, \mathbb{Y}\right\rangle_{B}=\sum_{k=1}^{m} \mu_{k}<\mathbf{X}^{k}, \mathbf{Y}^{k}>_{n},
$$

in which $<\mathbf{X}^{k}, \mathbf{Y}^{k}>_{n}=X_{1}^{k} Y_{1}^{k}+\ldots+X_{n}^{k} Y_{n}^{k}$ and where the weights $\mu_{1}, \ldots, \mu_{n}$ are defined by Eq. (8). The associated norm $\||\mathbb{X}|||$ of $\mathfrak{X}$ is then such that

$$
\|\mid \mathbb{X}\|\left\|^{2}=E\left\{\|\mathbb{X}\|_{B}^{2}\right\} \quad, \quad\right\| \mathbb{X}\left\|_{B}^{2}=\sum_{k=1}^{m} \mu_{k}\right\| \mathbf{x}^{k} \|_{n}^{2},
$$

in which $\left\|\mathbf{X}^{k}\right\|_{n}^{2}=\left(X_{1}^{k}\right)^{2}+\ldots+\left(X_{n}^{k}\right)^{2}$. For all $\mathbf{s}$ fixed in $\mathcal{S}$, we introduce the random vectors $\mathbb{W}(\mathbf{s})$ and $\mathbb{W}^{\text {exp }}$ such that

$$
\mathbb{W}(\mathbf{s})=\left(\mathbf{W}\left(\mathbf{s}, \omega_{1}\right), \ldots, \mathbf{W}\left(\mathbf{s}, \omega_{m}\right)\right), \mathbb{W}^{\exp }=\left(\mathbf{W}^{\exp }\left(\omega_{1}\right), \ldots, \mathbf{W}^{\exp }\left(\omega_{m}\right)\right) .
$$

Then the real vector $\underline{\mathbb{W}}^{\nu \exp }$ related to the experimental observations is introduced such that

$$
\underline{\mathbb{W}}^{\nu_{\exp }}=\frac{1}{\nu_{\exp }} \sum_{\ell=1}^{\nu_{\exp }} \mathbb{W}^{\exp }\left(\eta_{\ell}\right)
$$

On the other hand, the mean values $m(\mathbf{s})$ and $m^{\exp }$ of random vectors $\mathbb{W}(\mathbf{s})$ and $W^{\exp }$ are introduced such that

$$
\mathrm{m}(\mathbf{s})=E\{\mathbb{W}(\mathbf{s})\} \quad, \quad \mathrm{m}^{\mathrm{exp}}=E\left\{\mathbb{W}^{\mathrm{exp}}\right\}
$$

Equation (14) defined an estimation of $\mathrm{m}^{\mathrm{exp}}$ and if $\nu_{\exp }$ goes to infinity, then $\underline{\mathbb{W}}^{\nu^{\exp }}$ tends to $\mathrm{m}^{\exp }$ :

$$
\lim _{\nu_{\exp } \rightarrow+\infty} \underline{\mathbb{W}}^{\nu_{\exp }}=\mathrm{m}^{\exp }
$$

For all s fixed in $\mathcal{S}$, we introduce the following objective function $\mathbf{s} \mapsto \widetilde{J}_{\nu_{\exp }}(\mathbf{s})$ 
from $\mathcal{S}$ into $\mathbb{R}^{+}=[0,+\infty[$, such that

$$
\widetilde{J}_{\nu_{\exp }}(\mathbf{s})=\left\|\mathbb{W}(\mathbf{s})-\mathbb{W}^{\exp }\right\|^{2}
$$

The problem is to find $\mathbf{s}$ in $\mathcal{S}$ which minimizes $\widetilde{J}_{\nu_{\text {exp }}}$. Often the number $\nu_{\text {exp }}$ of experiments is not sufficiently large to be able to write $\mathrm{m}^{\exp } \simeq \underline{\mathbb{W}}^{\nu_{\exp }}$ and consequently, for such case we have

$$
E\left\{\mathbb{W}^{\exp }\right\}-\underline{\mathbb{W}}^{\nu_{\exp }}=\mathbb{b}_{\nu_{\exp }} \neq 0
$$

Clearly, vector $\mathbb{b}_{\nu_{\text {exp }}} \rightarrow 0$ when $\nu_{\text {exp }} \rightarrow+\infty$. We then consider that Eq. (18) holds, i.e., $\mathfrak{b}_{\nu_{\exp }} \neq 0$ for a given value of $\nu_{\text {exp }}$. Therefore Eq. (17) can be rewritten as

$$
\widetilde{J}_{\nu_{\text {exp }}}(\mathbf{s})=\| \mathbb{W}(\mathbf{s})-\mathrm{m}(\mathbf{s})-\mathbb{W}^{\exp }+\underline{\mathbb{W}}^{\nu_{\exp }}+\mathrm{m}(\mathbf{s})-\underline{\mathbb{W}}^{\nu^{\exp }}||^{2}
$$

Developing the right-hand side of Eq. (19), taking into account that the random variables $\mathbb{W}(\mathbf{s})-\mathrm{m}(\mathbf{s})$ and $\mathbb{W}^{\exp }-\underline{\mathbb{W}}^{\nu \text { exp }}$ are independent, removing the term $\left\|\left|\mathbb{W}^{\exp }-\underline{\mathbb{W}}^{\nu_{\exp }}\right|\right\|^{2}$ which is independent of $\mathbf{s}$, yield

$$
\begin{array}{r}
\widetilde{J}_{\nu_{\exp }}(\mathbf{s})=\|\| \mathbb{W}(\mathbf{s})-\mathrm{m}(\mathbf{s})\left\|^{2}+\right\| \mathrm{m}(\mathbf{s})-\underline{\mathbb{W}}^{\nu_{\exp }} \|_{B}^{2}+ \\
\ll \mathbb{W}^{\exp }-\underline{\mathbb{W}}^{\nu_{\exp }}, \mathrm{m}(\mathbf{s})-\underline{\mathbb{W}}^{\nu_{\exp }} \gg .
\end{array}
$$

It should be noted that the third term in the right-hand side of Eq. (20) can be rewritten as $<\mathfrak{b}_{\nu_{\exp }}, \mathrm{m}(\mathbf{s})-\underline{\mathbb{W}}^{\nu_{\exp }}>_{B}$. Consequently, from Eqs. (18) and (20), it can be deduced that

$$
\lim _{\nu_{\exp } \rightarrow+\infty} \widetilde{J}_{\nu_{\exp }}(\mathbf{s})=\widetilde{J}(\mathbf{s})
$$

in which the objective function $\mathbf{s} \mapsto \widetilde{J}(\mathbf{s})$ from $\mathcal{S}$ into $\mathbb{R}^{+}$is defined, for $\nu_{\text {exp }}$ sufficiently large (equivalently to $\nu_{\text {exp }} \rightarrow+\infty$ ) by

$$
\widetilde{J}(\mathbf{s})=\|\mathbb{W}(\mathbf{s})-\mathfrak{m}(\mathbf{s}) \mid\|^{2}+\left\|\mathfrak{m}(\mathbf{s})-\underline{\mathbb{W}}^{\nu_{\exp }}\right\|_{B}^{2} .
$$

Let us consider the identification of parameter $\mathbf{r}$ in $\mathcal{R} \subset \mathbb{R}^{n_{r}}$ with the mean computational model, i.e. with the stochastic computational model for $\delta=0$. We then have $\mathbf{s}_{0}=(\mathbf{r}, 0) \in \mathcal{S}$ and the optimization problem is written as

$$
\mathbf{s}^{\text {opt }}=\arg \min _{\mathbf{s}_{0}=(\mathbf{r}, 0) \in \mathcal{R} \times \Delta} \widetilde{J}\left(\mathbf{s}_{0}\right) \quad, \quad \widetilde{J}\left(\mathbf{s}_{0}\right)=\left\|\mathrm{m}\left(\mathbf{s}_{0}\right)-\underline{\mathbb{W}}^{\nu_{\exp }}\right\|_{B}^{2},
$$


in which $\mathbf{s}_{0}^{\text {opt }}=\left(\mathbf{r}^{\text {opt }}, 0\right)$. If $\widetilde{J}\left(\mathbf{s}_{0}^{\text {opt }}\right)=0$, then $\mathrm{m}\left(\mathbf{s}_{0}^{\text {opt }}\right)-\underline{\mathbb{W}}^{\nu_{\text {exp }}}=0$ and in mean, there is neither parameter uncertainties nor model uncertainties (note that with such an identified deterministic computational model, the dispersion of the experimental data induced by the variability of the real system is not taken into account). In general, $\widetilde{J}\left(\mathbf{s}_{0}^{\text {opt }}\right)$ is not equal to zero which means that there are data and model uncertainties which have to be considered in taking $\delta \neq 0$ and therefore the stochastic computational model must be used.

Considering the construction of the objective function, it is not necessary to use Eq. (20) or Eq. (22) and other objective functions can be derived. This is the way which is followed below.

\subsubsection{Case of a differentiable objective function and optimization problem}

Even if $\nu_{\text {exp }}$ is not sufficiently large, an objective function can be derived from Eq. (22) in replacing $\widetilde{J}$ by the following function $\mathbf{s} \mapsto J_{\gamma}^{D}(\mathbf{s})$ from $\mathcal{S}$ into $\mathbb{R}^{+}$ such that

$$
J_{\gamma}^{D}(\mathbf{s})=\left.2(1-\gamma)\|\mathbb{W}(\mathbf{s})-\mathrm{m}(\mathbf{s})\|\right|^{2}+2 \gamma\left\|\mathrm{m}(\mathbf{s})-\underline{\mathbb{W}}^{\nu_{\exp }}\right\|_{B}^{2},
$$

in which $\gamma$ is fixed in $] 0,1]$. In the right-hand side of Eq. (24), the first term is related to the variance of the stochastic computational model and the second one is related to the bias between the mean model and the mean value of experiments. The parameter $\gamma$ allows the balance between these two terms to be chosen and fixed. It should be noted that for all $\mathbf{s}$ in $\mathcal{S}, J_{1 / 2}^{D}(\mathbf{s})=\widetilde{J}(\mathbf{s})$. For a fixed value of $\gamma$, the identification of parameter $\mathbf{s}$ in $\delta$ is then the optimal value $\mathbf{s}^{\text {opt }}$ in $\mathcal{S}$ such that

$$
\mathbf{s}^{\mathrm{opt}}=\arg \min _{\mathbf{s} \in \mathcal{S}} J_{\gamma}^{D}(\mathbf{s})
$$

For $\gamma=0$, we have $J_{0}^{D}(\mathbf{s})=2\|\| \mathrm{W}(\mathbf{s})-\mathrm{m}(\mathbf{s}) \|\left.\right|^{2}$ and consequently, the optimization problem defined by Eq. (25) yields $\mathbf{s}^{\text {opt }}=\left(\mathbf{r}^{\text {opt }}, \delta^{\text {opt }}\right)$ with $\delta^{\text {opt }}=0$ and then $J_{0}^{D}\left(\mathbf{s}^{\mathrm{opt}}\right)=0$. Such a case is not coherent with the explanation given above and therefore, cannot be considered. This is the reason why the value $\gamma=0$ has to be removed from the admissible set $] 0,1]$ of values for $\gamma$.

For $\gamma=1$, we have $J_{1}^{D}(\mathbf{s})=2\left\|\mathrm{~m}(\mathbf{s})-\underline{\mathbb{W}}^{\nu_{\text {exp }}}\right\|_{B}^{2}$ and the optimization problem defined by Eq. (25) yields an optimal value $\mathbf{s}^{\text {opt }}$ which only minimizes the biais. In this case, the variance can take very high values and so the robustness of the prediction with the stochastic computational model decreases. Since the objective is to identify a stochastic computational model which is sufficiently robust with respect to uncertainties, then $\left\|\delta^{\text {opt }}\right\|$ has to be not too large and thus, the weight $2(1-\gamma)$ of the variance term in the objective function has to 
be increased with respect to the weight $2 \gamma$ of the biais term. Such a condition is satisfied in choosing $\gamma$ in $] 0, \frac{1}{2}[$.

In analyzing the objective function defined by Eq. (24), it can be seen that $\mathbf{s}^{\text {opt }}$ calculated with Eq. (25) does not take into account the dispersion of the experimental observations induced by the variabiliy of the real system but only uses the mean value of the experimental data. So if the dispersion of the experimental data around its mean value is important, then the objective function defined by Eq. (24) is not the most effective objective function. In this case, another objective function can be constructed in the context of the mean-square method and this question is treated in the following subsection in introducing a non-differentiable objective function.

\subsubsection{Case of a non-differentiable objective function and optimization prob- lem}

As explained in the previous subsection, this case is useful when the dispersion of the experimental observations is important. The criterion used to define the objective function is to write that, for all $\mathbf{s}$ in $\mathcal{S}$ and for all $j$ in $\{1, \ldots, n\}$, the experimental observations $\left\{W_{j}^{\exp }\left(\omega ; \eta_{\ell}\right), \omega \in B\right\}$ for all $\ell$ in $\left\{1, \ldots, \nu_{\text {exp }}\right\}$ belongs to the confidence region of the stochastic process $\left\{W_{j}(\mathbf{s}, \omega), \omega \in B\right\}$ associated with a probability level $\left.P_{c} \in\right] 0,1\left[\right.$. Let $\left\{w_{j}^{+}(\mathbf{s}, \omega), \omega \in B\right\}$ and $\left\{w_{j}^{-}(\mathbf{s}, \omega), \omega \in B\right\}$ be the upper and the lower envelopes which define the confidence region of stochastic process $\left\{W_{j}(\mathbf{s}, \omega), \omega \in B\right\}$ and such that, for all $\omega$ fixed in $B$, Proba $\left\{w_{j}^{-}(\mathbf{s}, \omega)<W_{j}(\mathbf{s}, \omega) \leq w_{j}^{+}(\mathbf{s}, \omega)\right\}=P_{c}$. We now define the functions $\left\{w_{j}^{+\exp }(\omega), \omega \in B\right\}$ and $\left\{w_{j}^{-\exp }(\omega), \omega \in B\right\}$ such that, for all $\omega$ in $B$, we have $w_{j}^{+\exp }(\omega)=\max _{\ell} W_{j}^{\exp }\left(\omega ; \eta_{\ell}\right)$ and $w_{j}^{-\exp }(\omega)=\min _{\ell} W_{j}^{\exp }\left(\omega ; \eta_{\ell}\right)$. The non-differentiable objective function is then constructed in order to minimize the area defined by the grey region shown in Fig. 1.

Using the sampling frequencies $\left\{\omega_{1}, \ldots, \omega_{m}\right\}$ of $B$, for all $k$ in $\{1, \ldots, m\}$, we then introduce the following two vectors $\mathbf{z}^{+}\left(\mathbf{s}, \omega_{k}\right)=\left(z_{1}^{+}\left(\mathbf{s}, \omega_{k}\right), \ldots, z_{n}^{+}\left(\mathbf{s}, \omega_{k}\right)\right)$ and $\mathbf{z}^{-}\left(\mathbf{s}, \omega_{k}\right)=\left(z_{1}^{-}\left(\mathbf{s}, \omega_{k}\right), \ldots, z_{n}^{-}\left(\mathbf{s}, \omega_{k}\right)\right)$ in $\mathbb{R}^{n}$ such that, for all $j$ in $\{1, \ldots, n\}$, we have

$z_{j}^{+}\left(\mathbf{s}, \omega_{k}\right)=\left(w_{j}^{+}\left(\mathbf{s}, \omega_{k}\right)-w_{j}^{+\exp }\left(\omega_{k}\right)\right)\left(1-\operatorname{Heav}\left(w_{j}^{+}\left(\mathbf{s}, \omega_{k}\right)-w_{j}^{+\exp }\left(\omega_{k}\right)\right)\right)$,

$z_{j}^{-}\left(\mathbf{s}, \omega_{k}\right)=\left(w_{j}^{-}\left(\mathbf{s}, \omega_{k}\right)-w_{j}^{-\exp }\left(\omega_{k}\right)\right)\left(1-\operatorname{Heav}\left(w_{j}^{-}\left(\mathbf{s}, \omega_{k}\right)-w_{j}^{-\exp }\left(\omega_{k}\right)\right)\right)$,

in which $x \mapsto \operatorname{Heav}(x)$ is the Heaviside function such that $\operatorname{Heav}(x)=1$ if $x \geq 0$ and Heav $(x)=0$ if $x<0$. Let $\mathbb{z}^{+}(\mathbf{s})$ and $\mathbb{z}^{-}(\mathbf{s})$ be the vectors in $\mathbb{R}^{n} \times \ldots \times \mathbb{R}^{n}$ ( $m$ times $) \simeq \mathbb{R}^{n m}$ such that

$$
\mathbb{Z}^{+}(\mathbf{s})=\left(\mathbf{z}^{+}\left(\mathbf{s}, \omega_{1}\right), \ldots, \mathbf{z}^{+}\left(\mathbf{s}, \omega_{m}\right), \mathbb{z}^{-}(\mathbf{s})=\left(\mathbf{z}^{-}\left(\mathbf{s}, \omega_{1}\right), \ldots, \mathbf{z}^{-}\left(\mathbf{s}, \omega_{m}\right) .\right.\right.
$$




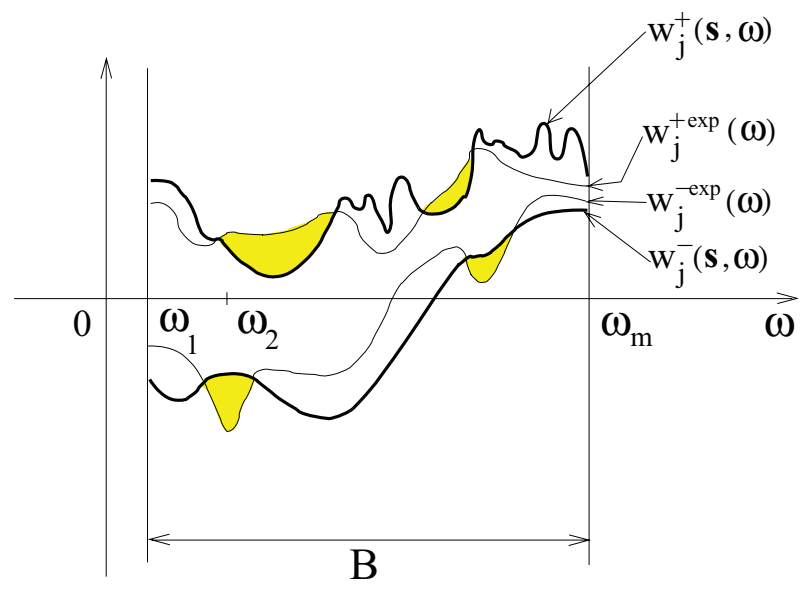

Fig. 1. For all $\mathbf{s}$ and for all $j$ fixed, the grey region represents the area minimized by the non-differentiable objective function. In the figure: $\left\{w_{j}^{+}(\mathbf{s}, \omega), \omega \in B\right\}$ and $\left\{w_{j}^{-}(\mathbf{s}, \omega), \omega \in B\right\}$ are the upper (upper thick solid line) and the lower (lower thick solid line) envelopes calulated with the stochastic computational model ; $\left\{w_{j}^{+\exp }(\omega), \omega \in B\right\}$ and $\left\{w_{j}^{-\exp }(\omega), \omega \in B\right\}$ are the upper (upper thin solid line) and the lower (lower thin solid line) envelopes for the experimental observations.

The objective function $\mathbf{s} \mapsto J^{N D}(\mathbf{s})$ from $\mathcal{S}$ into $\mathbb{R}^{+}$is then defined by

$$
J^{N D}(\mathbf{s})=\left\|\mathbb{Z}^{+}(\mathbf{s})\right\|_{B}^{2}+\left\|\mathbb{z}^{-}(\mathbf{s})\right\|_{B}^{2} .
$$

The objective function defined by Eq. (29) is not differentiable with respect to $\mathbf{s}$. The following optimization problem

$$
\mathbf{s}^{\text {opt }}=\arg \min _{\mathbf{s} \in \mathcal{S}} J^{N D}(\mathbf{s})
$$

allows the optimal value $\mathbf{s}^{\text {opt }}$ to be found in $\mathcal{S}$. Concerning the calculation of the upper and lower envelopes $\left\{w_{j}^{ \pm}\left(\mathbf{s}, \omega_{k}\right) ; j=1, \ldots, n ; k=1, \ldots, m\right\}$, the sample quantiles [55] is used. For all $j$ fixed in $\{1, \ldots, n\}$ and for all $k$ fixed in $\{1, \ldots, m\}$, let $F_{W_{j}\left(\mathbf{s}, \omega_{k}\right)}(w)=\operatorname{Proba}\left\{W_{j}\left(\mathbf{s}, \omega_{k}\right) \leq w\right\}$ be the cumulative distribution function which is assumed continuous from the right. For $0<p<$ 1 , the $p$ th quantile (or fractile) of $F_{W_{j}\left(\mathbf{s}, \omega_{k}\right)}$ is defined by

$$
\zeta(p)=\inf \left\{w: F_{W_{j}\left(\mathbf{s}, \omega_{k}\right)}(w) \geq p\right\} \quad .
$$

The lower and the upper envelopes for the symmetric interval are then defined by

$$
w_{j}^{+}\left(\mathbf{s}, \omega_{k}\right)=\zeta\left(\left(1+P_{c}\right) / 2\right) \quad, \quad w_{j}^{-}\left(\mathbf{s}, \omega_{k}\right)=\zeta\left(\left(1-P_{c}\right) / 2\right)
$$

Let $W_{j}\left(\mathbf{s}, \omega_{k} ; \theta_{1}\right), \ldots, W_{j}\left(\mathbf{s}, \omega_{k} ; \theta_{\nu}\right)$ be the $\nu$ independent realizations of random 
variable $W_{j}\left(\mathbf{s}, \omega_{k}\right)$. The estimation of the envelopes are written as

$$
\begin{array}{ll}
w_{j}^{+}\left(\mathbf{s}, \omega_{k}\right) \simeq \widetilde{w}_{j \ell^{+}}\left(\mathbf{s}, \omega_{k}\right) \quad, \quad \ell^{+}=\operatorname{fix}\left(\nu\left(1+P_{c}\right) / 2\right), \\
w_{j}^{-}\left(\mathbf{s}, \omega_{k}\right) \simeq \widetilde{w}_{j \ell^{-}}\left(\mathbf{s}, \omega_{k}\right) \quad, \quad \ell^{-}=\operatorname{fix}\left(\nu\left(1-P_{c}\right) / 2\right),
\end{array}
$$

in which $\operatorname{fix}(x)$ is the integer part of the real number $x$. In Eqs. (33) and $(34), \widetilde{w}_{j 1}\left(\mathbf{s}, \omega_{k}\right)<\ldots<\widetilde{w}_{j \nu}\left(\mathbf{s}, \omega_{k}\right)$ is the order statistics associated with $W_{j}\left(\mathbf{s}, \omega_{k} ; \theta_{1}\right), \ldots, W_{j}\left(\mathbf{s}, \omega_{k} ; \theta_{\nu}\right)$ which are calculated with the stochastic computational model using the Monte Carlo method.

\subsection{Maximum likelihood method}

In this subsection, we present the maximum likelihood method which allows the dispersion of the experimental data to be taken into account and which yields a more acurate estimation. However, the standard method requires a computational effort more important than the mean-square method. In order to decrease the computational effort, we will propose a statistical reduction of information.

For all $\mathbf{s}$ fixed in $\mathcal{S}$, let $\left(\mathbf{w}^{1}, \ldots, \mathbf{w}^{m}\right) \mapsto p\left(\mathbf{w}^{1}, \ldots, \mathbf{w}^{m}, \mathbf{s}\right)$ be the probability density function on $\mathbb{R}^{n} \times \ldots \times \mathbb{R}^{n}(m$ times $) \simeq \mathbb{R}^{n m}$ of the random observations $\left\{\mathbf{W}\left(\mathbf{s}, \omega_{1}\right), \ldots, \mathbf{W}\left(\mathbf{s}, \omega_{m}\right)\right\}$, defined by Eq. (9). The experimental observations are $\left\{\left\{\mathbf{W}^{\exp }\left(\omega_{1} ; \eta_{\ell}\right), \ldots, \mathbf{W}^{\exp }\left(\omega_{m} ; \eta_{\ell}\right)\right\}, \ell=1, \ldots, \nu_{\exp }\right\}$ defined by Eq. (10).

\subsubsection{Standard method}

Let $\mathbf{s} \mapsto \mathcal{L}(\mathbf{s})$ be the log-likelihood function from $\mathcal{S}$ into $\mathbb{R}$, defined by

$$
\mathcal{L}(\mathbf{s})=\sum_{\ell=1}^{\nu_{\text {exp }}} \log _{10} p\left(\mathbf{W}^{\exp }\left(\omega_{1} ; \eta_{\ell}\right), \ldots, \mathbf{W}^{\exp }\left(\omega_{m} ; \eta_{\ell}\right), \mathbf{s}\right)
$$

The maximum likelihood method $[64,66]$ consists in finding $\mathbf{s}^{\text {opt }}$ as the solution of the following optimization problem,

$$
\mathbf{s}^{\text {opt }}=\arg \max _{\mathbf{s} \in \mathcal{S}} \mathcal{L}(\mathbf{s})
$$

For all s fixed in $\mathcal{S}$, the right-hand side of Eq. (35) is estimated by using the stochastic computational model with the Monte Carlo method. If $m$ (the number of frequencies) is large, that is generally the case, the numerical cost can be very high and completely prohibitive. In order to decrease the numerical 
cost, function $\mathcal{L}(\mathbf{s})$ defined by Eq. (35) can be replaced as proposed in [2], [3] by

$$
\mathcal{L}(\mathbf{s})=\sum_{\ell=1}^{\nu_{\exp }} \sum_{k=1}^{m} \log _{10} p_{\mathbf{w}\left(\mathbf{s}, \omega_{k}\right)}\left(\mathbf{W}^{\exp }\left(\omega_{k} ; \eta_{\ell}\right), \mathbf{s}\right),
$$

in which $\mathbf{w}^{k} \mapsto p_{\mathbf{w}\left(s, \omega_{k}\right)}\left(\mathbf{w}^{k}, \mathbf{s}\right)$ is the probability density function on $\mathbb{R}^{n}$ with respect to $d \mathbf{w}^{k}$ of the $\mathbb{R}^{n}$-valued random variable $\mathbf{W}\left(\mathbf{s}, \omega_{k}\right)$. Such an approximation consists in replacing the joint probability density function of random variables $\mathbf{W}\left(\mathbf{s}, \omega_{1}\right), \ldots, \mathbf{W}\left(\mathbf{s}, \omega_{m}\right)$ by the product of the probability density function of each random variable $\mathbf{W}\left(\mathbf{s}, \omega_{k}\right)$. Clearly, such an approximation is exact if the random variables $\mathbf{W}\left(\mathbf{s}, \omega_{1}\right), \ldots, \mathbf{W}\left(\mathbf{s}, \omega_{m}\right)$ are mutually independent. In practice, such an assumption does not hold for dynamical systems which are considered in this paper and then, the statistical dependence is very important. As it can be seen in [2], [3], the use of Eq. (37) instead of Eq. (35) in the optimization problem defined by Eq. (36) can yield an optimal value $\mathbf{s}^{\text {opt }}$ for which the dispersion parameter $\delta$ is overestimated, that is to say, for which the values of the components of $\delta$ are too large (such an approximation removes the statistical dependence and therefore, is too conservative). In the next subsection, we propose another approximation which corresponds to a good compromise between Eq. (35) and Eq. (37).

\subsubsection{Statistical reduction of information}

The use of Eq. (37) instead of Eq. (35) would be better if the random vectors $\mathbf{W}\left(\mathbf{s}, \omega_{1}\right), \ldots, \mathbf{W}\left(\mathbf{s}, \omega_{m}\right)$ were not correlated (even if these vectors stay mutually dependent). Unfortunately, random vectors $\mathbf{W}\left(\mathbf{s}, \omega_{1}\right), \ldots, \mathbf{W}\left(\mathbf{s}, \omega_{m}\right)$ are generally correlated. The idea is then to proceed to a statistical reduction using a principal component analysis (see for instance [32]) and then to use the maximum likelihood method in the space of the uncorrelated random variables related to the reduced statistical information.

For all $\mathbf{s}$ fixed in $\mathcal{S}$, let $\mathbb{W}(\mathbf{s})$ be the $\mathbb{R}^{n m}$-valued random variable defined by Eq. (13) and let $m(\mathbf{s}) \in \mathbb{R}^{n m}$ be its mean value defined by Eq. (15). Let $\left[C_{\mathbb{W}}(\mathbf{s})\right]$ be the $(n m \times n m)$ covariance matrix defined by

$$
\left[C_{\mathbb{W}}(\mathbf{s})\right]=E\left\{(\mathbb{W}(\mathbf{s})-\mathrm{m}(\mathbf{s}))(\mathbb{W}(\mathbf{s})-\mathfrak{m}(\mathbf{s}))^{T}\right\} .
$$

Note that $\mathrm{m}(\mathbf{s})$ and $\left[C_{\mathbb{W}}(\mathbf{s})\right]$ can easily be estimated by using the stochastic computational model with the Monte Carlo method. We introduce the eigenvalue problem

$$
\left[C_{\mathbb{W}}(\mathbf{s})\right]_{\mathfrak{x}}(\mathbf{s})=\lambda(\mathbf{s}) \times(\mathbf{s}),
$$


for which the positive eigenvalues are such that $\lambda_{1}(\mathbf{s}) \geq \lambda_{2}(\mathbf{s}) \geq \ldots$. The corresponding eigenvectors $x^{1}(\mathbf{s}), \mathfrak{x}^{2}(\mathbf{s}), \ldots$ belong to $\mathbb{R}^{n m}$ and are written as $\mathbb{x}^{\alpha}(\mathbf{s})=\left(\mathbf{x}^{\alpha 1}(\mathbf{s}), \ldots, \mathbf{x}^{\alpha m}(\mathbf{s})\right) \in \mathbb{R}^{n} \times \ldots \times \mathbb{R}^{n}(m$ times $) \simeq \mathbb{R}^{n m}$. These vectors constitute an orthonormal family such that $<x^{\alpha}(\mathbf{s}), \mathrm{x}^{\beta}(\mathbf{s})>_{B}=\delta_{\alpha \beta}$ (Kronecker symbol). Let $N$ be an integer such that $1 \leq N<n m$. We introduce the approximation $\mathbb{W}^{N}(\mathbf{s})$ of $\mathbb{W}(\mathbf{s})$ defined by

$$
\mathbb{W}^{N}(\mathbf{s})=\mathfrak{m}(\mathbf{s})+\sum_{\alpha=1}^{N} \sqrt{\lambda_{\alpha}(\mathbf{s})} Y_{\alpha}(\mathbf{s}) \varkappa^{\alpha}(\mathbf{s})
$$

in which $Y_{1}(\mathbf{s}), \ldots, Y_{N}(\mathbf{s})$ are $N$ real-valued random variables such that

$$
Y_{\alpha}(\mathbf{s})=\frac{1}{\sqrt{\lambda_{\alpha}(\mathbf{s})}}<\mathbb{W}(\mathbf{s})-\mathrm{m}(\mathbf{s}), \varkappa^{\alpha}(\mathbf{s})>_{B} \quad, \quad \forall \alpha=1, \ldots, N
$$

Let $\mathbf{Y}(\mathbf{s})=\left(Y_{1}(\mathbf{s}), \ldots, Y_{N}(\mathbf{s})\right)$ be the $\mathbb{R}^{N}$-valued random variable. It can easily be proven that $\mathbf{Y}(\mathbf{s})$ is a second-order random variable such that, for all $\alpha$ and $\beta$ in $\{1, \ldots, N\}$,

$$
E\left\{Y_{\alpha}(\mathbf{s})\right\}=0 \quad, \quad E\left\{Y_{\alpha}(\mathbf{s}) Y_{\beta}(\mathbf{s})\right\}=\delta_{\alpha \beta},
$$

which means that the centered random variables $Y_{1}(\mathbf{s}), \ldots, Y_{N}(\mathbf{s})$ are uncorrelated. The order $N$ of the statistical reduction is calculated in order to get an approximation with a given accuracy $\varepsilon$, independent of $N$ and $\mathbf{s}$, such that, for all $\mathbf{s}$ in $\mathcal{S}$,

$$
\left\|\left|W(\mathbf{s})-\mathbb{W}^{N}(\mathbf{s})\left\|\left.\right|^{2}=\varepsilon\right\|\right||W(\mathbf{s}) \||^{2}\right.
$$

Since $\left\|\mathbb{W}(\mathbf{s})-\mathbb{W}^{N}(\mathbf{s})\right\|^{2}=\operatorname{tr}\left[C_{\mathbb{W}}(\mathbf{s})\right]-\sum_{\alpha=1}^{N} \lambda_{\alpha}(\mathbf{s})$ in which tr is the trace of matrix, $N$ has to be chosen such that

$$
\max _{\mathbf{s} \in \mathcal{S}}\left(1-\frac{\sum_{\alpha=1}^{N} \lambda_{\alpha}(\mathbf{s})}{\operatorname{tr}\left[C_{\mathbb{W}}(\mathbf{s})\right]}\right) \leq \varepsilon
$$

In practice, for all $\mathbf{s}$ fixed in $\mathcal{S}$, the dominant subspace of matrix $\left[C_{\mathbb{W}}(\mathbf{s})\right]$ associated with the $N$ first eigenvalues $\lambda_{1}(\mathbf{s}) \geq \ldots \geq \lambda_{N}(\mathbf{s})$ is calculated by using an iteration algorithm such as the subspace iteration method. The statistical reduction will be efficient if $N \ll n m$.

Let $\left(y_{1}, \ldots, y_{N}\right) \mapsto p_{Y_{1}(\mathbf{s}), \ldots, Y_{N}(\mathbf{s})}\left(y_{1}, \ldots, y_{N}, \mathbf{s}\right)$ be the probability density function on $\mathbb{R}^{N}$ with respect to $d y_{1} \ldots d y_{N}$ of the random variables $Y_{1}(\mathbf{s}), \ldots, Y_{N}(\mathbf{s})$. 
It should be noted that, if the random variables $Y_{1}(\mathbf{s}), \ldots, Y_{N}(\mathbf{s})$ are uncorrelated by construction (see Eq. (42)), in general, these random variables are mutually dependent. From Eq. (41), it can be deduced that, for all $\alpha$ in $\{1, \ldots, N\}$ and for all $\ell$ in $\left\{1, \ldots, \nu_{\exp }\right\}$, the experimental realization $Y_{\alpha}^{\exp }\left(\mathbf{s}, \eta_{\ell}\right)$ is given by

$$
Y_{\alpha}^{\exp }\left(\mathbf{s}, \eta_{\ell}\right)=\frac{1}{\sqrt{\lambda(\mathbf{s})}}<\mathbb{W}^{\exp }\left(\eta_{\ell}\right)-\mathrm{m}(\mathbf{s}), \mathrm{x}^{\alpha}(\mathbf{s})>_{B} \quad, \quad \forall \alpha=1, \ldots, N .
$$

It should be noted that the experimental generalized coordinate $Y_{\alpha}^{\exp }\left(\mathbf{s}, \eta_{\ell}\right)$ depends on s. Let $\mathbf{s} \mapsto \mathcal{L}^{\text {red }}(\mathbf{s})$ be the log-likelihood function from $\mathcal{S}$ into $\mathbb{R}$, defined for all $\mathbf{s}$ fixed in $\mathcal{S}$ by

$$
\mathcal{L}^{\mathrm{red}}(\mathbf{s})=\sum_{\ell=1}^{\nu_{\exp }} \log _{10} p_{Y_{1}(\mathbf{s}), \ldots, Y_{N}(\mathbf{s})}\left(Y_{1}^{\exp }\left(\mathbf{s}, \eta_{\ell}\right), \ldots, Y_{N}^{\exp }\left(\mathbf{s}, \eta_{\ell}\right), \mathbf{s}\right)
$$

The maximum likelihood method yields the optimal value $\mathbf{s}^{\text {opt }}$ in $\mathcal{S}$ of $\mathbf{s}$,

$$
\mathbf{s}^{\text {opt }}=\arg \max _{\mathbf{s} \in \mathcal{S}} \mathcal{L}^{\text {red }}(\mathbf{s})
$$

If $N$ is not too large, then Eq. (46) can be used to solve the optimization problem defined by Eq. (47). If $N$ is still sufficiently large such that Eq. (46) induces a prohibitive numerical cost, a similar approximation to the one introduced by Eq. (37) can be used. Therefore, the log-likelihood function $\mathcal{L}^{\text {red }}$ can be replaced by the following one,

$$
\mathcal{L}^{\mathrm{red}}(\mathbf{s})=\sum_{\ell=1}^{\nu_{\exp }} \sum_{\alpha=1}^{N} \log _{10} p_{Y_{\alpha}(\mathrm{s})}\left(Y_{\alpha}^{\exp }\left(\mathbf{s}, \eta_{\ell}\right), \mathbf{s}\right)
$$

in which $y \mapsto p_{Y_{\alpha}(\mathrm{s})}(y, \mathbf{s})$ is the probability density function on $\mathbb{R}$ with respect to $d y$ of the real-valued random variable $Y_{\alpha}(\mathbf{s})$. The introduction of such an approximation consists in writting the log-likelihood function $\mathcal{L}^{\text {red }}$ as if the random variables $Y_{1}(\mathbf{s}), \ldots, Y_{N}(\mathbf{s})$ were mutually independent, that is not true. Nevertheless, this approximation is better than for Eq. (37) because random variables $Y_{1}(\mathbf{s}), \ldots, Y_{N}(\mathbf{s})$ are uncorrelated (see Eq. (42)).

The values of the density $p_{Y_{1}(\mathbf{s}), \ldots, Y_{N}(\mathbf{s})}\left(Y_{1}^{\exp }\left(\mathbf{s}, \eta_{\ell}\right), \ldots, Y_{N}^{\exp }\left(\mathbf{s}, \eta_{\ell}\right), \mathbf{s}\right)$ for the experimental values, or the values of the densities $p_{Y_{\alpha}(\mathrm{s})}\left(Y_{\alpha}^{\exp }\left(\mathbf{s}, \eta_{\ell}\right), \mathbf{s}\right)$ for the experimental values are performed with the stochastic computational model and the Monte Carlo method. Note that the $\nu$ independent realizations $Y_{1}\left(\mathbf{s}, \theta_{1}\right), \ldots$, $Y_{N}\left(\mathbf{s}, \theta_{N}\right)$ are calculated using Eq. (41),

$$
Y_{\alpha}\left(\mathbf{s}, \theta_{\ell}\right)=\frac{1}{\sqrt{\lambda_{\alpha}(\mathbf{s})}}<\mathbb{W}\left(\mathbf{s}, \theta_{\ell}\right)-\mathrm{m}(\mathbf{s}), \mathrm{k}^{\alpha}(\mathbf{s})>_{B} \quad, \quad \forall \alpha=1, \ldots, N,
$$


and that Eq. (45) is used to calculate the projection of the experimental observations.

\subsection{Comments on the optimization algorithms}

The optimization problems defined by Eqs. (25), (30), (36) or (47) have to be solved with appropriate algorithms. Note that the objective functions defined above are not convex functions. In addition, since these optimization problems are related to the admissible set $\mathcal{S}$, this set has to be described in terms of inequalities and linear and/or nonlinear constraint equations. Three main cases can be considered:

(1) Except for the mean-square method with a non-differentiable objective function, deterministic algorithms using the gradient of the objective function can be used (see for instance $[11,12,26,28,48,66,64]$ ).

(2) For the mean-square method with a non-differentiable objective function, the genetic algorithm (see for instance $[13,14,27,64]$ ) or the stochastic search methods (see [64]) such as the pattern search algorithms (see for instance $[1,39])$ can be used.

(3) If dimension $n_{s}$ of parameter $\mathbf{s}$ is very low (for instance $n_{s}=1,2$ or 3 ), the graph of the objective function can directly be constructed for a finite partition of $\delta$.

\section{Applications and experimental validation}

In this Section, we present different applications with experimental validations covering all the cases developed in Section 5 The first Subsection 6.1 is devoted to structural dynamics and the second Subsection 6.2 deals with structural acoustics.

\subsection{Structural dynamics}

Subsection 6.1 deals with the construction of the mean computational model and the stochastic reduced model of structures in linear dynamics. These elements will be used in the two applications presented in Subsections 6.1.1 and 6.1.2 which are devoted to the identification of the mean model parameters and of the stochastic model parameters using experimental data. The dynamical system is made up of a damped elastic structure. The usual formulation 
in displacement (see for instance [44]) is used and the mean computational model of the dynamical system is constructed with the finite element method. Let $\underline{\mathbf{u}}(\omega)$ be the $\mathbb{C}^{n_{s}}$-vector of the $n_{s}$ dofs of the structure. Let $\left\{\underline{\varphi}_{1}, \ldots, \underline{\varphi}_{N_{s}}\right\}$ be the $N_{s}$ first elastic modes of the structure at zero frequency (and thus not including the rigid body modes). The reduced mean model is obtained by projection of the mean computational model on the subspace spanned by $\left\{\underline{\varphi}_{1}, \ldots, \underline{\varphi}_{N_{s}}\right\}$ (see for instance [44]). The reduced mean model can then be written, for all $\omega$ fixed in the frequency band of analysis $B=\left[\omega_{0}, \omega_{1}\right]$ with $0<\omega_{0}<\omega_{1}$, as

$$
\underline{\mathbf{u}}(\omega)=\sum_{\alpha=1}^{N_{s}} \underline{q}_{\alpha}^{s}(\omega) \underline{\varphi}_{\alpha} .
$$

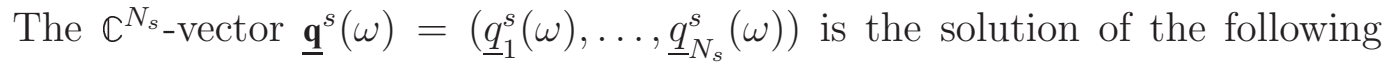
matrix equation

$$
\left(-\omega^{2}\left[\underline{M}_{s}\right]+i \omega\left[\underline{D}_{s}(\omega)\right]+\left[\underline{K}_{s}(\omega)\right]\right) \underline{\mathbf{q}}^{s}(\omega)=\underline{\mathbf{f}}^{s}(\omega) \quad .
$$

In the above equations, the $\left(N_{s} \times N_{s}\right)$ real matrices $\left[\underline{M}_{s}\right],\left[\underline{D}_{s}(\omega)\right]$ and $\left[\underline{K}_{s}(\omega)\right]$ are the generalized mass, damping and stiffness matrices of the structure. The generalized damping and stiffness matrices are assumed to be dependent on the frequency due to viscoelastic effects. The $\mathbb{C}^{N_{s}}$-vector $\underline{\mathbf{f}}^{s}(\omega)$ is the generalized force vector of the structure.

The use of the nonparametric probabilistic approach [57] to [62] of both model uncertainties and parameter uncertainties in the structure consists in modeling the generalized mass $\left[\underline{M}_{s}\right]$, damping $\left[\underline{D}_{s}(\omega)\right]$ and stiffness $\left[\underline{K}_{s}(\omega)\right]$ matrices of the structure by random matrices $\left[\mathbf{M}_{s}\right],\left[\mathbf{D}_{s}(\omega)\right]$ and $\left[\mathbf{K}_{s}(\omega)\right]$ whose dispersion parameters are denoted by $\delta_{M_{s}}, \delta_{D_{s}}$ and $\delta_{K_{s}}$ respectively and are independent of dimension $N_{s}$ and frequency $\omega$. The explicit construction of the probability distribution of these random matrices is given in [57],[58],[62]. Let $[\mathbf{H}(\omega)]$ be anyone of these random matrices (independent of $\omega$ for random matrix $\left[\mathbf{M}_{s}\right]$ ). The probability distribution of such a random matrix $[\mathbf{H}(\omega)]$ depends only on its mean value $[\underline{H}(\omega)]=E\{[\mathbf{H}(\omega)]\}$ and on its dispersion parameter $\delta_{H}$ which is independent of $N_{s}$ and $\omega$. An algebraic representation of random matrix $[\mathbf{H}(\omega)]$ has been developed and allows independent realizations to be constructed for a stochastic solver based on the Monte Carlo numerical simulation. Since $[\underline{H}(\omega)]$ is a symmetric positive-definite real matrix, then the random matrix $[\mathbf{H}(\omega)]$ is written as $[\mathbf{H}(\omega)]=\left[\underline{L}_{H}(\omega)\right]^{T}[\mathbf{G}]\left[\underline{L}_{H}(\omega)\right]$ in which $[\underline{H}(\omega)]=\left[\underline{L}_{H}(\omega)\right]^{T}\left[\underline{L}_{H}(\omega)\right]$ and where the random matrix germ $[\mathbf{G}]$ is independent of $\omega$. The stochastic reduced model of the uncertain dynamical system for which the reduced mean 
model is defined by Eqs. (50) and (51) is written, for all $\omega$ fixed in $B$, as

$$
\underline{\mathbf{U}}(\omega)=\sum_{\alpha=1}^{N_{s}} \underline{Q}_{\alpha}^{s}(\omega) \underline{\varphi}_{\alpha}
$$

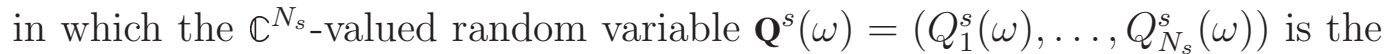
solution of the following random matrix equation

$$
\left(-\omega^{2}\left[\mathbf{M}_{s}\right]+i \omega\left[\mathbf{D}_{s}(\omega)\right]+\left[\mathbf{K}_{s}(\omega)\right]\right) \underline{\mathbf{Q}}^{s}(\omega)=\underline{\mathbf{f}}^{s}(\omega) \quad .
$$

\subsubsection{Low- and medium-frequency vibration of a composite sandwich panel}

This subsection deals with an application devoted to the identification of the mean model parameters and of the stochastic model parameters using experimental data for low- and -medium frequency vibration of a composite sandwich panel. The designed composite sandwich panel is made up of five layers made of four thin carbon-resin unidirectional plies and one high stiffness closed-cell foam core. The dimensions of the panel are $0.40 \mathrm{~m}$ length, $0.30 \mathrm{~m}$ width and $0.01068 \mathrm{~m}$ total thickness. Eight sandwich panels $\left(\nu_{\text {exp }}=8\right)$ have been manufactured from the designed sandwich panel using an identical process and the same materials. All the sandwich panels have been baked in the same batch for suppressing the influences of the different baking conditions concerning time and temperature. The panel is vertical and suspended by two thin soft rubber bands attached to the two upper corners of the panel. The frequency band of analysis is $B=[150,4500] \mathrm{Hz}$. The number of sampling frequencies is $m=584$ with a frequency resolution $\Delta f$ decreasing when frequency is increasing. The eigenfrequency of the vertical body motion is about $2 \mathrm{~Hz}$ which has to be compared to the lowest elastic eigenfrequency of the panel which is $191 \mathrm{~Hz}$. Consequently, the measurements of the frequency response functions in the frequency band of analysis are performed for a configuration corresponding to free-free conditions. This structure and the experiments are completely defined in [9], [10].

The input force is a point load normal to the panel applied to a given point such that all the symmetric and anti-symmetric elastic modes of the panel are excited in the frequency band of analysis. The outputs are made up of normal accelerations to the panel measured at 24 points. The observation of the structure (see Eq. (6) and Section 4) is then the real vector $\mathbf{W}(\mathbf{s}, \omega)$ in $\mathbb{R}^{n}$ with $n=24$ and where $W_{j}(\mathbf{s}, \omega)=20 \log _{10}\left(\omega^{2}\left|V_{k_{j}}(\mathbf{s}, \omega)\right|\right)$ in which $\omega^{2}\left|V_{k_{1}}(\mathbf{s}, \omega)\right|, \ldots, \omega^{2}\left|V_{k_{n}}(\mathbf{s}, \omega)\right|$ are the moduli of the normal accelerations measured at the 24 points in the panel. For each experimental panel $\eta_{\ell}$ with $\ell=1, \ldots, \nu_{\exp }$, the corresponding experimental observation is $\mathbf{W}^{\exp }\left(\omega ; \eta_{\ell}\right)$ in which $W_{j}^{\exp }\left(\omega ; \eta_{\ell}\right)=20 \log _{10}\left(\omega^{2}\left|V_{k_{j}}^{\exp }(\omega)\right|\right)$. Below, the optimization problems 
are solved using the 24 measured accelerations but the results are shown only for two points in the panel in order to limit the number of figures. One of the two points $(j=3)$ is located close to an edge of the panel and the other one $(j=17)$ is close to the input force.

The mean computational model is a finite element model with 12, 288 structural dofs $(64 \times 64$ four-nodes finite elements for laminated plate bending). This mean computational model has been updated with respect to the mass density of each carbon-resine ply and to the Young moduli of each carbon-resin ply in order to update the eigenfrequecies of the first four elastic modes using the experimental values (see [9], [10]). Below, this updated mean computational model will simply be called the mean computational model. The reduced mean computational model is constructed with $N_{s}=180$ elastic modes and with a constant mean damping rate $\xi=0.01$. Figs. 2 and 3 compare the experimental measurements $\omega \mapsto W_{k_{3}}^{\exp }\left(\omega ; \eta_{\ell}\right)$ and $\omega \mapsto W_{k_{17}}^{\exp }\left(\omega ; \eta_{\ell}\right)$ for $\ell=1, \ldots, 8$ (the 8 experimental panels) with $\omega \mapsto \underline{w}_{k_{3}}(\omega)$ and $\omega \mapsto \underline{w}_{k_{17}}(\omega)$ calculated with the mean computational model. These two figures show that there are significant

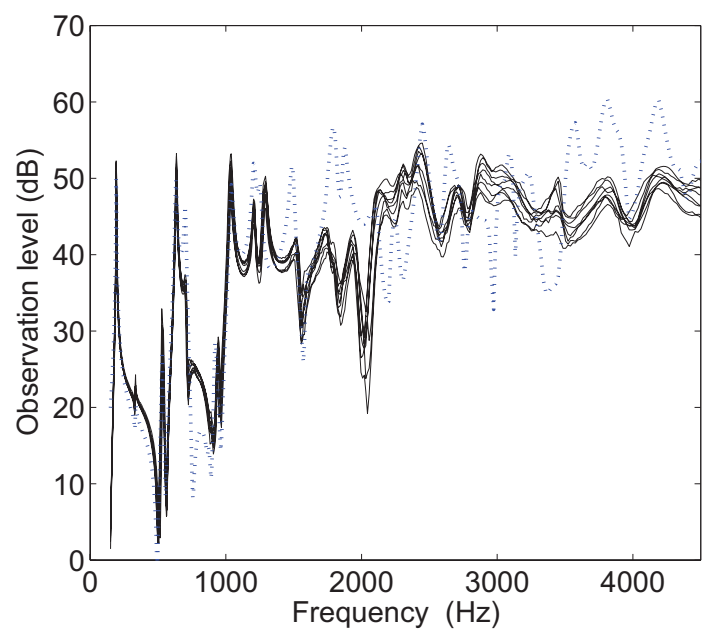

Fig. 2. Comparison of the 8 experimental measurements for observation 3 ( 8 thin solid lines) with the mean computational model (dotted line).

differences in the medium-frequency band $[1500,4500] \mathrm{Hz}$ which are mainly induced by the mean damping model used in the mean computational model and by model uncertainties. Consequently, it is necessary to proceed to an identification of the mean damping model in presence of model uncertainties.

Firstly, we introduce an algebraic representation of the generalized damping matrix (of the mean computational model) depending on a vector-valued parameter $\mathbf{r}$ which has to be identified. The generalized damping matrix of the mean computational model is rewritten [7] as the following positive-definite 


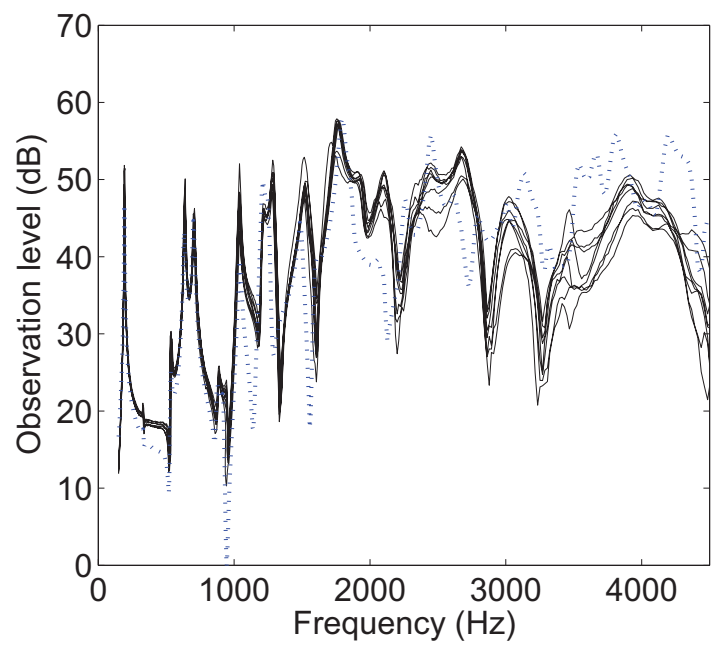

Fig. 3. Comparison of the 8 experimental measurements for observation 17 ( 8 thin solid lines) with the mean computational model (dotted line).

diagonal $\left(N_{s} \times N_{s}\right)$ real matrix $\left[\underline{D}_{s}(\mathbf{r}, \omega)\right]$ such that

$$
\left[\underline{D}_{s}(\mathbf{r}, \omega)\right]_{\alpha \alpha}=2 \xi\left(\mathbf{r}, \omega_{\alpha}\right) \mu_{\alpha} \omega_{\alpha}, \xi(\mathbf{r}, \omega)=\xi_{0}+\left(\xi_{1}-\xi_{0}\right) \frac{\omega^{a}}{\omega^{a}+10^{b}}
$$

in which $\mathbf{r}=\left(\xi_{0}, \xi_{1}, a, b\right) \in\left(\mathbb{R}^{+}\right)^{4}$ with $\xi_{0} \leq \xi_{1}$ and where $\mu_{\alpha}$ and $\omega_{\alpha}$ are the generalized mass and the eigenfrequency of elastic mode $\alpha$. Secondly, the stochastic reduced computational model is introduced with dispersion parameters $\delta_{M_{s}}, \delta_{D_{s}}$ and $\delta_{K_{s}}$ for the generalized mass, damping and stiffness matrices. Thirdly, the vector-valued identification parameter $\mathbf{s}=(\mathbf{r}, \boldsymbol{\delta})$ is introduced such that $\mathbf{r}=\left(\xi_{0}, \xi_{1}, a, b\right)$ and $\delta=\left(\delta_{M_{s}}, \delta_{D_{s}}, \delta_{K_{s}}\right)$. We then have $n_{r}=4$ and $n_{\delta}=3$ and $n_{s}=7$. The admissible set $\delta \subset \mathbb{R}^{n_{s}}$ is then defined by the constraints $0.0095 \leq \xi_{0} \leq 0.0105,0.05 \leq \xi_{1} \leq 0.15,5 \leq a \leq 20,30 \leq b \leq 50$, $0.05 \leq \delta_{M_{s}} \leq 0.5,0.05 \leq \delta_{D_{s}} \leq 0.5$ and $0.05 \leq \delta_{K_{s}} \leq 0.5$.

For the experimental indentification of parameter $\mathbf{s} \in \mathcal{S}$, the mean-square method (see Section 5.2) is used with two different objective functions. The first one is the differentiable objective function defined by Eq. (24) with $\gamma=0.25$ and the optimization problem defined by Eq. (25) is solved by using the Sequential Quadratic Optimization Algorithm (see Section 5.4 (1)) with constraints defining the admissible set $\mathcal{S}$. The second one is the nondifferentiable objective function defined by Eq. (29) and the optimization problem defined by Eq. (30) is solved by using a Genetic Algorithm (see Section $5.4(2)$ ) with constraints defining $\mathcal{S}$. To calculate the values of the objectives functions, the Monte Carlo simulation is performed with $\nu=400$ independent realizations (corresponding to a reasonable mean-square convergence).

Figs. 4 to 7 display, for observations $j=3$ and $j=17$, the comparisons be- 
tween experimental measurements $\left(f \mapsto W_{k_{j}}^{\exp }\left(2 \pi f, \eta_{\ell}\right)\right)$ and the predictions calculated (1) with the mean computational model $\left(f \mapsto \underline{w}_{k_{j}}(2 \pi f)\right),(2)$ with the optimal mean computational model $\left(f \mapsto \underline{w}_{k_{j}}\left(\mathbf{r}^{\mathrm{opt}}, 2 \pi f\right)\right.$ and (3) with the optimal stochastic computational model $\left(f \mapsto W_{k_{j}}\left(\mathbf{s}^{\mathrm{opt}}, 2 \pi f\right)\right)$ for the two objective functions (differentiable and non-differentiable objective functions).

Figs. 4 and 5 are related to observation 3 and 17 for differentiable objective function and correspond to $\mathbf{s}^{\mathrm{opt}}=\left(\mathbf{r}^{\mathrm{opt}}, \delta^{\mathrm{opt}}\right)$ with $\mathbf{r}^{\mathrm{opt}}=(0.01,0.085,10.7,46.1)$ and $\boldsymbol{\delta}=(0.31,0.20,0.14)$. Figs. 6 and 7 are related to observation 3 and 17 for non-differentiable objective function and correspond to $\mathbf{s}^{\mathrm{opt}}=\left(\mathbf{r}^{\mathrm{opt}}, \delta^{\mathrm{opt}}\right)$ with $\mathbf{r}^{\text {opt }}=(0.01,0.081,10.9,47)$ and $\delta=(0.23,0.07,0.24)$. These figures have been constructed with $\nu=1000$ independent realizations. The confidence regions are constructed with a probability level $P_{c}=0.95$. It can be seen that (1) the identification performed with both objective functions considerably increases the quality of the predictions in the medium-frequency range with respect to the initial mean computational model, (2) the non-differentiable objective function is more effective than the differentiable function but the numerical cost is greater for the non-differentiable objective function due to the use of the genetic algorithm.

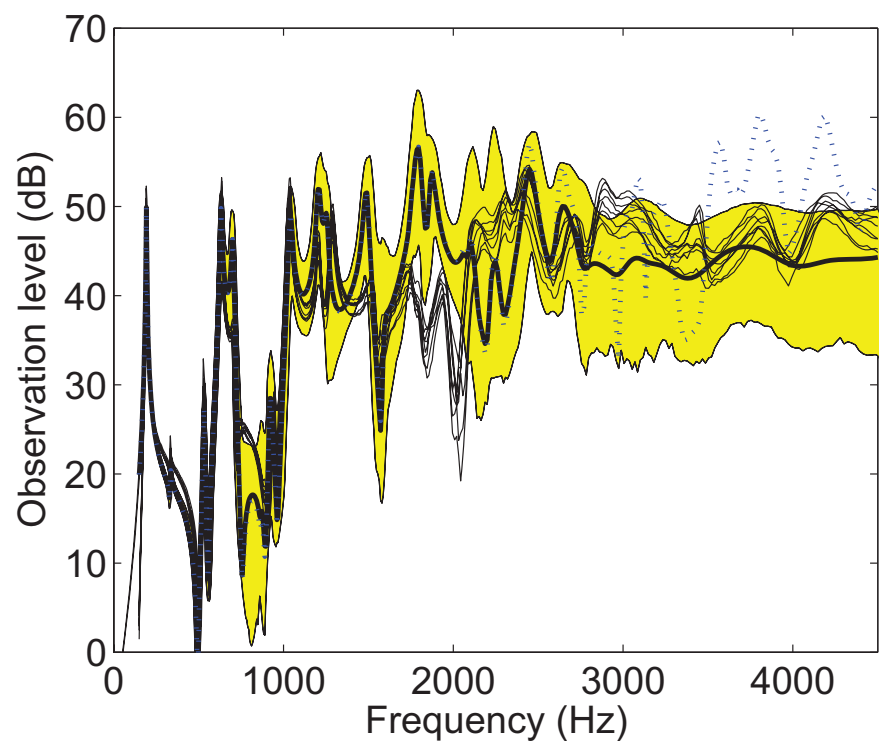

Fig. 4. Differentiable objective function, observation 3. Comparison of the 8 experimental measurements ( 8 thin solid lines) with (1) the mean computational model (dotted line), (2) the optimal mean computational model (thick solid line), (3) the confidence region of the optimal stochastic computational model (grey region). 


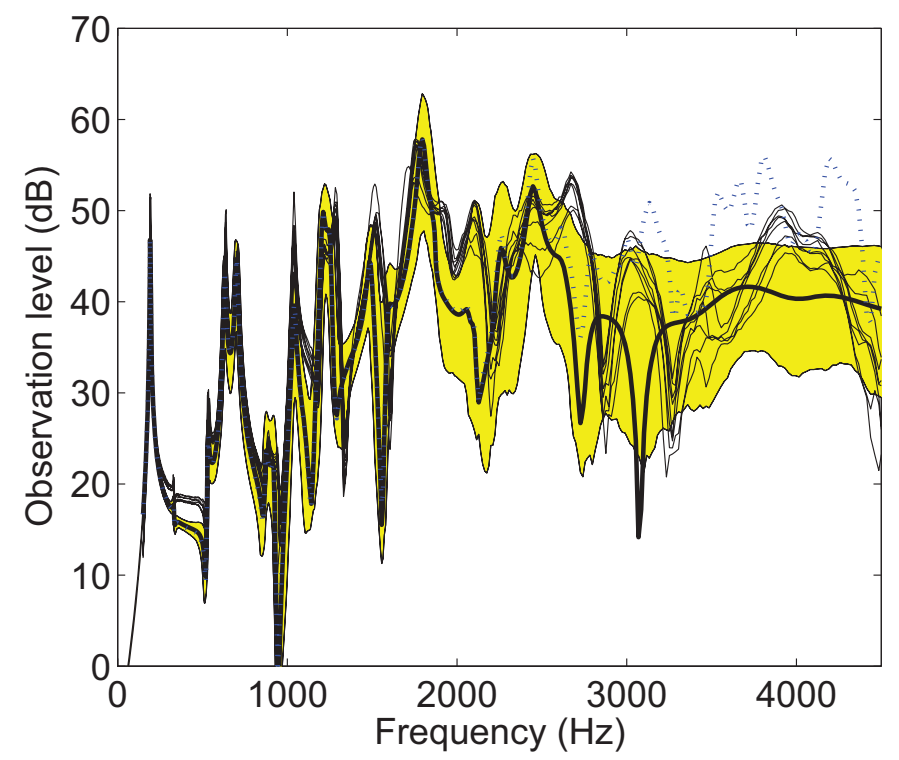

Fig. 5. Differentiable objective function, observation 17. Comparison of the 8 experimental measurements ( 8 thin solid lines) with (1) the mean computational model (dotted line), (2) the optimal mean computational model (thick solid line), (3) the confidence region of the optimal stochastic computational model (grey region).

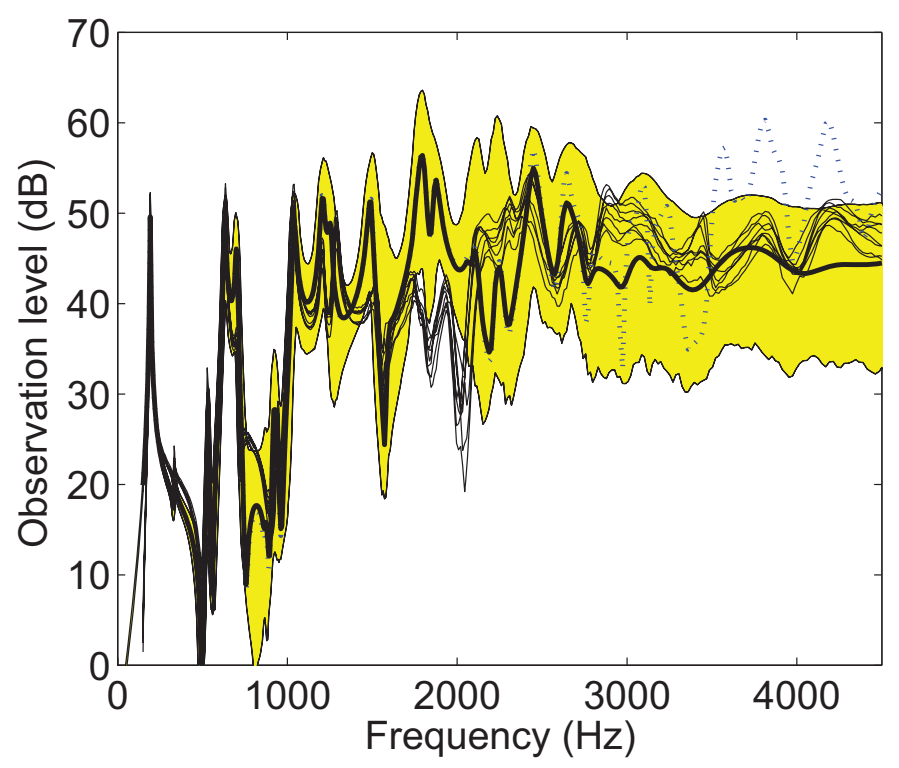

Fig. 6. Non-differentiable objective function, observation 3. Comparison of the 8 experimental measurements ( 8 thin solid lines) with (1) the mean computational model (dotted line), (2) the optimal mean computational model (thick solid line), (3) the confidence region of the optimal stochastic computational model (grey region).

\subsubsection{Low-frequency vibration of a thin plate connected to a framework}

This subsection deals with an application devoted to the identification (using experimental data) of the dispersion parameters of the nonparametric 


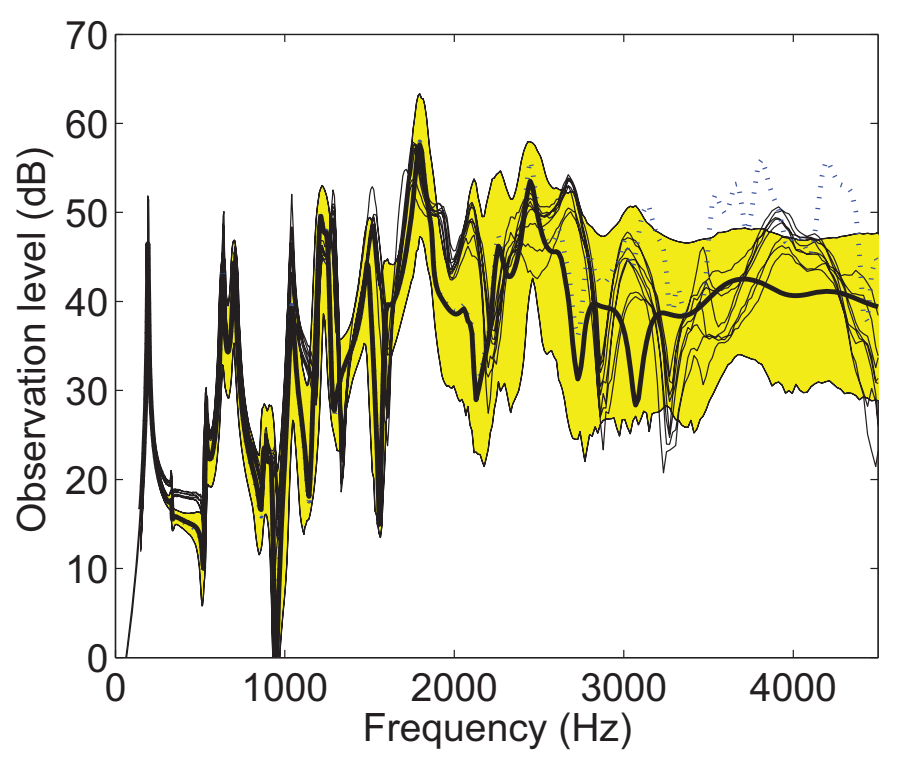

Fig. 7. Non-differentiable objective function, observation 17. Comparison of the 8 experimental measurements ( 8 thin solid lines) with (1) the mean computational model (dotted line), (2) the optimal mean computational model (thick solid line), (3) the confidence region of the optimal stochastic computational model (grey region).

probabilistic approach of model uncertainties for low-frequency vibration of a structure in linear dynamics. The structure is made up of a homogeneous, isotropic and slightly damped thin plate (steel plate with a constant thickness) connected to an elastic framework on its edges. This dynamical system is hung by four springs and then there are no rigid body modes. The highest eigenfrequency of suspension is $9 \mathrm{~Hz}$ while the lowest eigenfrequency of the elastic modes is $43 \mathrm{~Hz}$. The excitation is a point force applied to the framework and exciting the dynamical system mainly in bending mode in the frequency band of analysis which is $B=] 0,250] \mathrm{Hz}$. The number of sampling frequencies is $m=250$. The frequency resolution is $\Delta f=1 \mathrm{~Hz}$. Only one experiment $\left(\nu_{\text {exp }}=1\right)$ has been performed for this structure. The frequency response functions have been measured on band $B$ for 60 normal accelerations in the plate. A complete description of the experimental test can be found in [22]. The observation (see Eq. (6) and Section 4) is the real quantity $W(\mathbf{s}, \omega)=$ $10 \log _{10}\left(\sum_{i=1}^{60}\left(\omega^{2}\left|V_{k_{i}}(\mathbf{s}, \omega)\right|\right)^{2}\right)$ in which $\omega^{2}\left|V_{k_{1}}(\mathbf{s}, \omega)\right|, \ldots, \omega^{2}\left|V_{k_{60}}(\mathbf{s}, \omega)\right|$ are the moduli of the normal accelerations measured at the 60 points in the plate (we then have $n=1)$. The corresponding experimental observation is $W^{\exp }(\omega)=$ $10 \log _{10}\left(\sum_{i=1}^{60}\left(\omega^{2}\left|V_{k_{i}}^{\exp }(\omega)\right|\right)^{2}\right)$.

The mean computational model is a finite element model having 16, 104 structural dofs. The reduced mean computational model is constructed with $N_{s}=$ 139 structural modes. The mean computational model has been updated with respect to the Young modulus and the mass density of the plate and of the framework using the experimental values of the two first elastic modes and the 
ninth elastic mode (first elastic torsion mode of the structure). The updated mean computational model will simply be called below the mean computational model. Fig. 8 compares the experimental measurements $\omega \mapsto W^{\exp }(\omega)$ with the result $\omega \mapsto \underline{w}(\omega)$ given by the mean computational model. It can be seen that the mean computational model yields a good prediction in the frequency band $] 0,80 \mathrm{Jz}$ while model uncertainties are present for the frequency band $[80,250] \mathrm{Hz}$. Consequently, the nonparametric probabilistic approach of data and model uncertainties is used to improve the prediction and the dispersion parameters are identified with the experiments. We introduce the

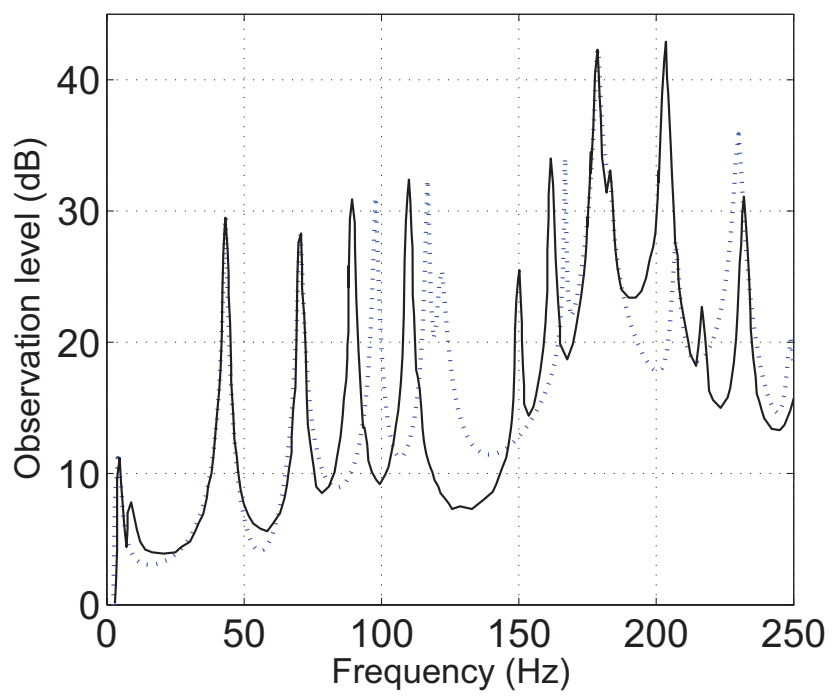

Fig. 8. Comparison of the experimental measurement (solid line) with the result given by the mean computational model (dotted line).

real-valued identification parameter $s=\delta$ related to the dispersion parameter which is such that $\delta=\delta_{M_{s}}=\delta_{K_{s}}$. the dispersion parameter $\delta_{D_{s}}$ related to the generalized damping matrix of the structure is taken as $\delta_{D_{s}}=0.3$. The Monte Carlo simulation is performed with $\nu=10,000$ independent realizations (mean-square convergence is reached for 1000 realizations). The mean-square convergence of the solution of the stochastic reduced model is reached for $N_{s}=80$ for all the values of $\delta$.

The maximum likelihood with the statistical reduction of information (see Section 5.3.2) is used. As we have explained in Section 5.3, the use of the standard method would lead us to an overestimate of the dispersion parameters and therefore is not adapted (note that the standard method would yield a value which would be about three times the value estimated with the statistical reduction; this factor has effectively been calculated with the standard method for the present application). Consequently, we have to solve the optimization problem defined by Eq. (47) for which the objective function is defined by Eq. (48) with $\nu_{\text {exp }}=1$ and $N$ defined hereinafter. Fig. 9 displays the graphs of the function $N \mapsto\left(1-\sum_{\alpha=1}^{N} \lambda_{\alpha}(s) / \operatorname{tr}\left[C_{\mathbb{W}}(s)\right]\right)^{1 / 2}$ related to Eq. (44) for 12 val- 
ues of the parameter $s=\delta=0.05,0.10,0.15,0.18,0.20,0.25,0.30,0.35,0.40$, $0.45,0.50,0.65)$. This figure shows that the nominal value of $N$ is 90 corresponding to a relative error $\varepsilon=0.047$. Fig. 10 displays the graph of the function $\delta \mapsto \mathcal{L}^{\text {red }}(\delta)$ for $\delta$ belonging to the interval [0.05, 0.65]. This figure shows that the optimal value $\delta^{\text {opt }}$ which is the solution of the optimization problem defined by Eq. (47) is such that $\delta^{o p t}=0.25$. Fig. 11 shows the comparaison of the experimental measurements $f \mapsto W^{\exp }(2 \pi f)$ with the prediction of the confidence region calculated with the optimal stochastic reduced model $\left(f \mapsto W\left(\delta^{\mathrm{opt}}, 2 \pi f\right)\right.$ for a probability level of 0.95 .

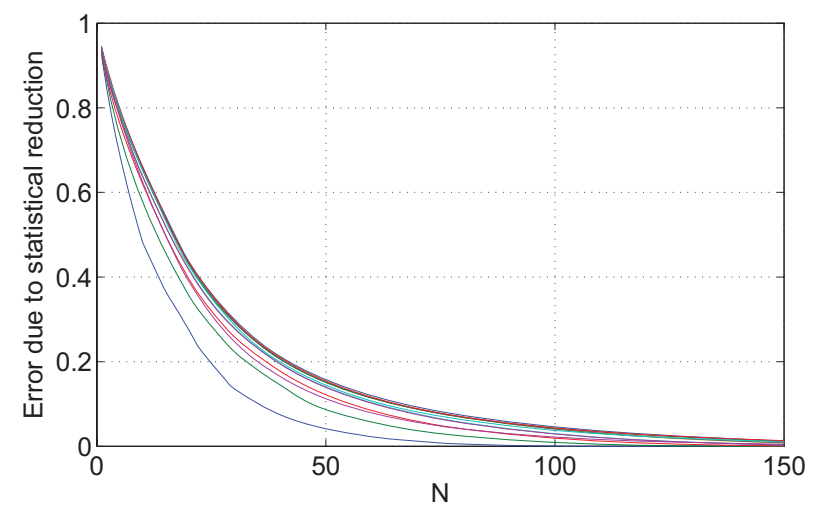

Fig. 9. Graphs of the error as a function of statistical reduction dimension $N$ for 12 values of $s=\delta$.

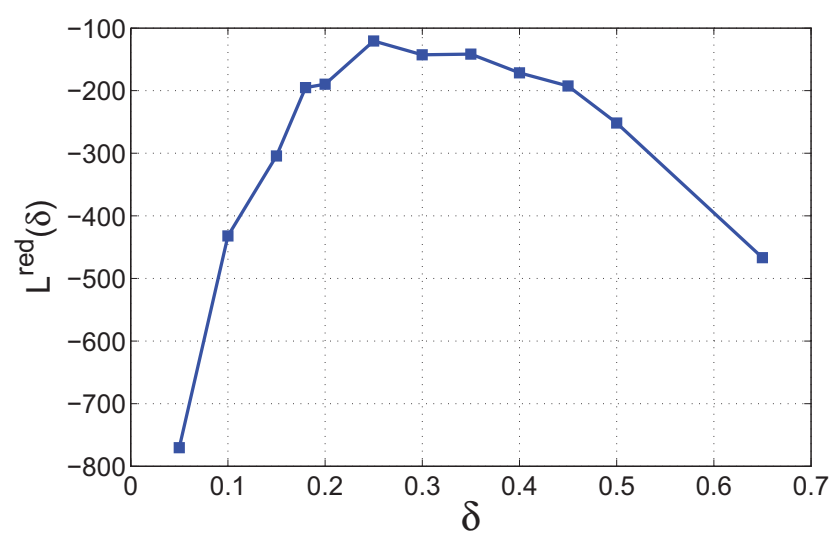

Fig. 10. Graph of function $\delta \mapsto \mathcal{L}^{\text {red }}(\delta)$.

\subsection{Structural acoustics}

In this section, we present an application devoted to the identification of the dispersion parameters of the nonparametric probabilistic approach of model uncertainties for vibroacoustic analysis of a car using experimental data [19,20]. 


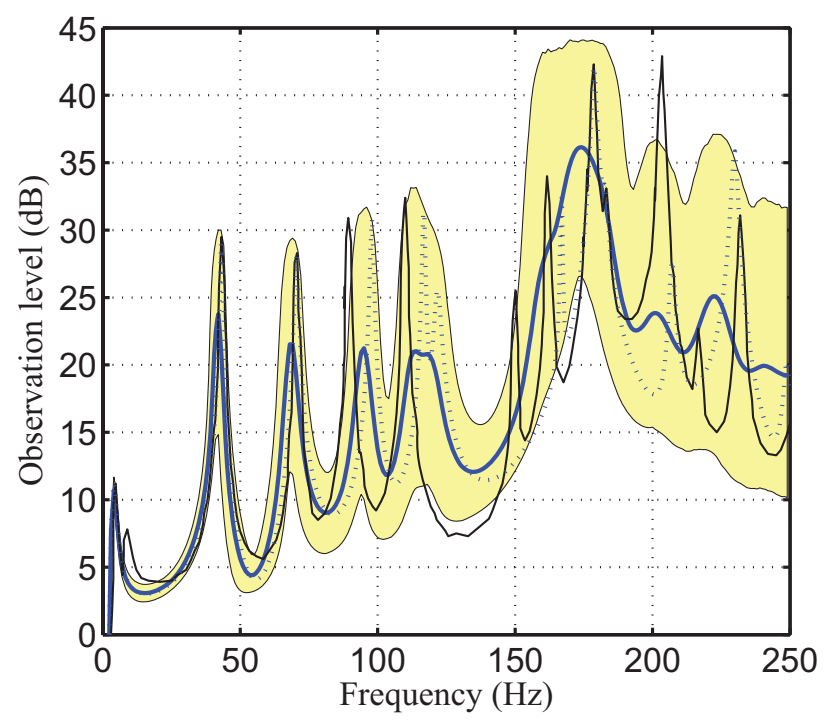

Fig. 11. Comparison of the experimental measurements (thin solid line) with (1) the updated computational model (dotted line), (2) with the mean value of optimal stochastic reduced model (thick solid line), (3) with the confidence region calculated with the optimal stochastic reduced model (grey region).

The structural acoustic system is made up of a damped structure coupled with an internal acoustic cavity filled with a dissipating acoustic fluid. The usual formulation in "structural displacement" - "acoustic pressure" (see Ref. [44]) is used and the mean computational model of the structural acoustic system is constructed with the finite element method. Let $\underline{\mathbf{u}}(\omega)$ be the $\mathbb{C}^{n_{s}}$-vector of the $n_{s}$ dofs of the structure and let $\underline{\mathbf{p}}(\omega)$ be the $\mathbb{C}^{n_{f}}$-vector corresponding to the $n_{f}$ dofs of the acoustic cavity. Let $\left\{\underline{\varphi}_{1}, \ldots, \underline{\varphi}_{N_{s}}\right\}$ be the $N_{s}$ first structural modes of the structure in vacuo and calculated at zero frequency (not including rigid body modes if there exist). Let $\left\{\underline{\boldsymbol{\psi}}_{1}, \ldots, \underline{\boldsymbol{\psi}}_{N_{f}}\right\}$ be the $N_{f}$ first acoustic modes of the acoustic cavity with rigid fluid-structure coupling interface (including the constant pressure mode, the acoustic cavity being assumed to be closed). The reduced mean model is obtained by projection of the mean finite element model on the subspaces spanned by $\left\{\underline{\boldsymbol{\varphi}}_{1}, \ldots, \underline{\boldsymbol{\varphi}}_{N_{s}}\right\}$ and $\left\{\underline{\boldsymbol{\psi}}_{1}, \ldots, \underline{\boldsymbol{\psi}}_{N_{f}}\right\}$. The reduced mean model can then be written as

$$
\underline{\mathbf{u}}(\omega)=\sum_{\alpha=1}^{N_{s}} \underline{q}_{\alpha}^{s}(\omega) \underline{\varphi}_{\alpha} \quad, \quad \underline{\mathbf{p}}(\omega)=\sum_{\beta=1}^{N_{f}} \underline{q}_{\beta}^{f}(\omega) \underline{\boldsymbol{\psi}}_{\beta} .
$$

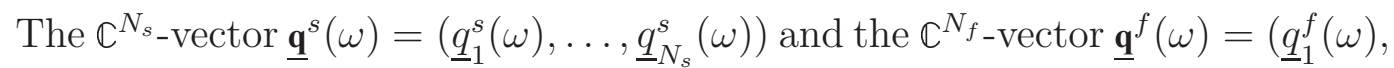
$\left.\ldots, \underline{q}_{N_{f}}^{f}(\omega)\right)$ are the solution of the following matrix equation

$$
\left[\begin{array}{cc}
{\left[\underline{a}_{s}(\omega)\right]} & {[\underline{C}]} \\
\omega^{2}[\underline{C}]^{T} & {\left[\underline{a}_{f}(\omega)\right]}
\end{array}\right]\left[\begin{array}{l}
\underline{\mathbf{q}}^{s}(\omega) \\
\underline{\mathbf{q}}^{f}(\omega)
\end{array}\right]=\left[\begin{array}{l}
\underline{\mathbf{f}}^{s}(\omega) \\
\underline{\mathbf{f}}^{f}(\omega)
\end{array}\right]
$$


in which $\left[\underline{a}_{s}(\omega)\right]=-\omega^{2}\left[\underline{M}_{s}\right]+i \omega\left[\underline{D}_{s}\right]+\left[\underline{K}_{s}\right]$ and where $\left[\underline{a}_{f}(\omega)\right]=-\omega^{2}\left[\underline{M}_{f}\right]+$ $i \omega\left[\underline{D}_{f}\right]+\left[\underline{K}_{f}\right]$. In the above equations, the $\left(N_{s} \times N_{s}\right)$ real matrices $\left[\underline{M}_{s}\right]$, $\left[\underline{D}_{s}\right]$ and $\left[\underline{K}_{s}\right]$ are the generalized mass, damping and stiffness matrices of the structure, the $\left(N_{f} \times N_{f}\right)$ real matrices $\left[\underline{M}_{f},\left[\underline{D}_{f}\right]\right.$ and $\left[\underline{K}_{f}\right]$ are the generalized mass, damping and stiffness matrices of the acoustic and where the rectangular $\left(N_{s} \times N_{f}\right)$ real matrix $[\underline{C}]$ is the generalized vibroacoustic coupling matrix.

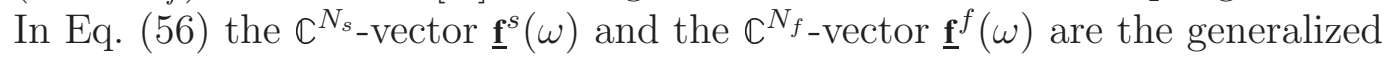
force vector of the structure and the generalized acoustic source vector of the acoustic cavity respectively.

The use of the nonparametric probabilistic approach [57] to [62] of both model uncertainties and parameter uncertainties for the structure, the acoustic cavity and the vibroacoustic coupling consists (1) in modeling the generalized mass $\left[\underline{M}_{s}\right]$, damping $\left[\underline{D}_{s}\right]$ and stiffness $\left[\underline{K}_{s}\right]$ matrices of the structure by random matrices $\left[\mathbf{M}_{s}\right],\left[\mathbf{D}_{s}\right]$ and $\left[\mathbf{K}_{s}\right]$ whose dispersion parameters are $\delta_{M_{s}}, \delta_{D_{s}}$ and $\delta_{K_{s}}$ respectively; (2) in modeling the generalized mass $\left[\underline{M}_{f}\right]$, damping $\left[\underline{D}_{f}\right]$ and stiffness $\left[\underline{K}_{f}\right]$ matrices of the acoustic cavity by random matrices $\left[\mathbf{M}_{f}\right],\left[\mathbf{D}_{f}\right]$ and $\left[\mathbf{K}_{f}\right]$ whose dispersion parameters are $\delta_{M_{f}}, \delta_{D_{f}}$ and $\delta_{K_{f}}$ respectively; (3) in modeling the generalized vibroacoustic coupling matrix $[\underline{C}]$ by a random matrix $[\mathbf{C}]$ whose dispersion parameter is $\delta_{C}$. The explicit construction of the probability distribution of these random matrices is given in [57], [58], [62] for random matrices $\left[\mathbf{M}_{s}\right],\left[\mathbf{D}_{s}\right],\left[\mathbf{K}_{s}\right],\left[\mathbf{M}_{f}\right],\left[\mathbf{D}_{f}\right]$ and $\left[\mathbf{K}_{f}\right]$, and is given in [62] for random matrix $[\mathbf{C}]$. Let $[\mathbf{H}]$ be anyone of these random matrices. The probability distribution of such a random matrix $[\mathbf{H}]$ depends only on its mean value $[\underline{H}]=E\{[\mathbf{H}]\}$ and on its dispersion parameter $\delta_{H}$ which must be taken independent of the matrix dimension. An algebraic representation of random matrix $[\mathbf{H}]$ has been developed and allows independent realizations to be constructed for a stochastic solver based on the Monte Carlo numerical simulation. For random matrices $\left[\mathbf{M}_{s}\right],\left[\mathbf{D}_{s}\right],\left[\mathbf{K}_{s}\right],\left[\mathbf{M}_{f}\right],\left[\mathbf{D}_{f}\right]$ and $\left[\mathbf{K}_{f}\right]$, random matrix $[\mathbf{H}]$ is then a symmetric positive-definite (or positive) real-valued random matrix and $[\mathbf{H}]$ is written as $[\mathbf{H}]=\left[\underline{L}_{H}\right]^{T}\left[\mathbf{G}_{H}\right]\left[\underline{L}_{H}\right]$ in which $[\underline{H}]=\left[\underline{L}_{H}\right]^{T}\left[\underline{L}_{H}\right]$ and where $\left[\mathbf{G}_{H}\right]$ is the random matrix germ. When $[\mathbf{H}]$ is the rectangular matrix $[\mathbf{C}]$ (see $[62]$ ), using the following polar decomposition $[\underline{C}]=[\underline{U}][\underline{T}]$ with $[\underline{U}]^{T}[\underline{U}]=[I]$ and where $[\underline{T}]$ is a positive-definite matrix which can then be factorized as $[\underline{T}]=\left[\underline{L}_{C}\right]^{T}\left[\underline{L}_{C}\right]$, random matrix $[\mathbf{C}]$ is written as $[\mathbf{C}]=\left[\underline{U}^{\prime}\right]\left[\underline{L}_{C}\right]^{T}\left[\mathbf{G}_{C}\right]\left[\underline{L}_{C}\right]$ in which $\left[\mathbf{G}_{C}\right]$ is another random matrix germ. The stochastic reduced model of the uncertain structural acoustic system for which the reduced mean model is defined by Eqs. (55) and (56) is written, for all $\omega$ fixed in the frequency band of analysis $B=\left[\omega_{0}, \omega_{1}\right]$ with $0<\omega_{0}<\omega_{1}$, as

$$
\underline{\mathbf{U}}(\omega)=\sum_{\alpha=1}^{N_{s}} \underline{Q}_{\alpha}^{s}(\omega) \underline{\varphi}_{\alpha} \quad, \quad \underline{\mathbf{P}}(\omega)=\sum_{\beta=1}^{N_{f}} \underline{Q}_{\beta}^{f}(\omega) \underline{\boldsymbol{\varphi}}_{\beta}
$$




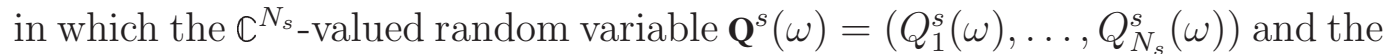

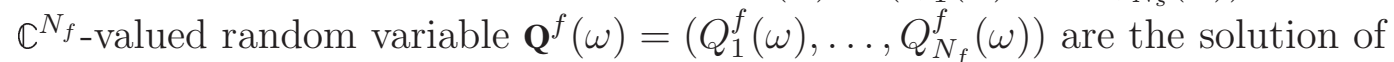
the following random matrix equation

$$
\left[\begin{array}{cc}
{\left[\mathbf{A}_{s}(\omega)\right]} & {[\mathbf{C}]} \\
\omega^{2}[\mathbf{C}]^{T} & {\left[\mathbf{A}_{f}(\omega)\right]}
\end{array}\right]\left[\begin{array}{l}
\mathbf{Q}^{s}(\omega) \\
\mathbf{Q}^{f}(\omega)
\end{array}\right]=\left[\begin{array}{l}
\underline{\mathbf{f}}^{s}(\omega) \\
\underline{\mathbf{f}}^{f}(\omega)
\end{array}\right]
$$

in which the random complex matrices $\left[\mathbf{A}_{s}(\omega)\right]$ and $\left[\mathbf{A}_{f}(\omega)\right]$ are defined by $\left[\mathbf{A}_{s}(\omega)\right]=-\omega^{2}\left[\mathbf{M}_{s}\right]+i \omega\left[\mathbf{D}_{s}\right]+\left[\mathbf{K}_{s}\right]$ and where $\left[\mathbf{A}_{f}(\omega)\right]=-\omega^{2}\left[\mathbf{M}_{f}\right]+i \omega\left[\mathbf{D}_{f}\right]+\left[\mathbf{K}_{f}\right]$.

The mean computational vibroacoustic model of the car is a finite element model with 978,733 structural dofs and 8,139 acoustic pressure dofs in the internal acoustic cavity. The finite element mesh of the structure is shown in Fig. 12 and the finite element mesh of the acoustic cavity is shown in Fig. 13. There are $n=6$ observed accelerations located in the points $O 1$ to $O 6$ of the structure and are shown in Fig. 14. The external applied forces are point forces applied to the engine supports. The reduced mean computational model in constructed using $N_{s}=1,723$ elastic modes of the structure in vacuo and $N_{f}=57$ acoustic modes of the internal acoustic cavity with rigid walls. Such dimensions of the reduced model yield convergence of the stochastic solution. The experimental database has been constructed using $\nu_{\text {exp }}=20$ cars of the same type with different optional extra. The experimental structural observations are the accelerations measured at the 6 observed points $O 1$ to $O 6$ shown in Fig. 14. The mean-square method with differentiable objective

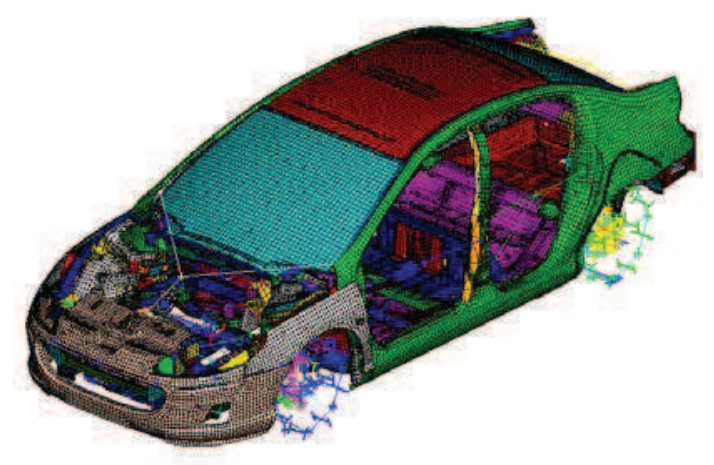

Fig. 12. Finite element mesh of the structure: 978,733 structural dofs.

function is used. Consequently, we have to solve the optimization problem defined by Eq. (25) for which the objective function is defined by Eq. (24) with $\gamma=0.5$. In this objective function, frequency band of analysis is defined by $B=[100,180] \mathrm{Hz}$ with a frequency resolution equal to $0.5 \mathrm{~Hz}$. Parameter $\mathbf{r}$ is fixed to the nominal value used in the mean computational model. The dispersion parameter $\delta_{D_{s}}$ related to the generalized damping matrix of the structure in the mean computational model and the dispersion parameter $\delta_{C}$ related to the vibroacoustic coupling interface have been fixed to given value 


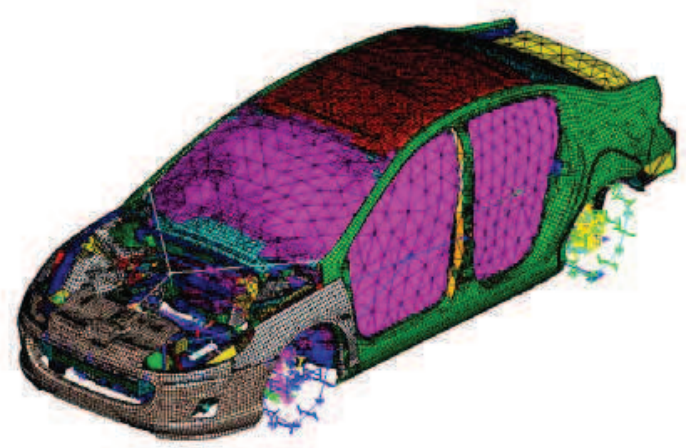

Fig. 13. Finite element mesh of the acoustic cavity: 8, 139 acoustic pressure dofs.

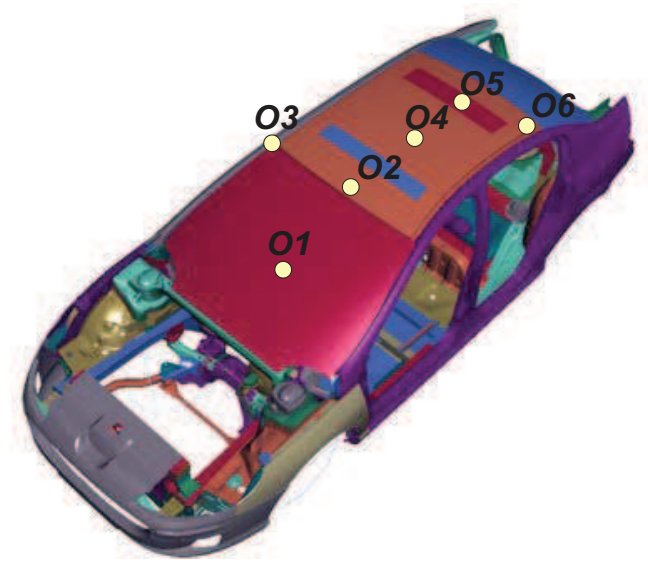

Fig. 14. Definition of the observations $O 1$ to $O 6$ of the structure.

denoted as $\delta_{D_{s}}^{\text {opt }}$ and $\delta_{C}^{\text {opt }}$. In a first step, the dispersion parameters $\delta_{M_{f}}, \delta_{D_{f}}$ and $\delta_{K_{f}}$ related to the acoustic cavity have been identified using acoustic experiments and the maximum likelihood method (see [19]). The optimal values obtained are denoted by $\delta_{M_{f}}^{\mathrm{opt}}, \delta_{D_{f}}^{\mathrm{opt}}$ and $\delta_{K_{f}}^{\mathrm{opt}}$. In a second step, the parameter $\mathbf{s}=\boldsymbol{\delta}=\left(\delta_{M_{s}}, \delta_{K_{s}}\right)$ is identified. In Eq. (24), the random observation vector $\mathbb{W}(\mathbf{s})$ with values in $\mathbb{R}^{n}$ is made up of the observed degrees of freedom which are normal accelerations to the structure in logarithmic scale (see Section 2, point (3)). For each evaluation of the objective function, the stochastic reduced computational model is solved using the Monte Carlo method with a number of independent realizations equal to $\nu=1000$ and corresponding to a mean-square convergence of the second-order stochastic solution. Since each evaluation of the objective function requires about 500 hours of CPU time (the computations have been realized with $20 \mathrm{CPU}$ yielding an elapsed time of 25 hours for each evaluation of the objective function), we have limited the computational effort to solve the optimization problem in directly constructing the graph of function $\delta \mapsto j(\delta)$ for 10 values of $\delta$ (see Fig. 15) in which $j(\delta)=J_{1 / 2}^{D}(\mathbf{s})$ with $\delta=\sqrt{\delta_{M_{s}}^{2}+\delta_{K_{s}}^{2}}$. Fig. 15 displays the graph of function $\delta \mapsto j(\delta)$ and shows that there is a minimum in the admissible set of values 


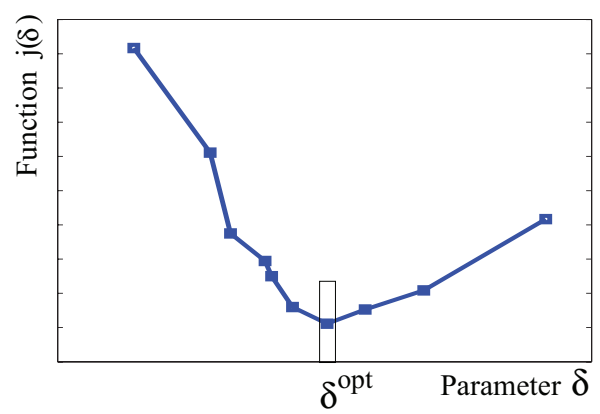

Fig. 15. Graph of the objective function $\delta \mapsto j(\delta)$

corresponding to the optimal value $\delta^{\text {opt }}$ of $\delta$. In the trial method used, this optimal value $\delta^{\text {opt }}$ of $\delta$ yields a corresponding optimal value $\left(\delta_{M_{s}}^{\mathrm{opt}}, \delta_{K_{s}}^{\mathrm{opt}}\right)$ of $\left(\delta_{M_{s}}, \delta_{K_{s}}\right)$ for which $\delta_{M_{s}}^{\mathrm{opt}} \neq \delta_{K_{s}}^{\mathrm{opt}}$. A complete calculation has been performed with the optimal stochastic reduced computational model for the values $\delta_{M_{s}}^{\mathrm{opt}}, \delta_{D_{s}}^{\mathrm{opt}}, \delta_{K_{s}}^{\mathrm{opt}}$, $\delta_{M_{f}}^{\mathrm{opt}}, \delta_{D_{f}}^{\mathrm{opt}}, \delta_{K_{f}}^{\mathrm{opt}}$ and $\delta_{C}^{\mathrm{opt}}$. Fig. 16 compares the experimental measurements with

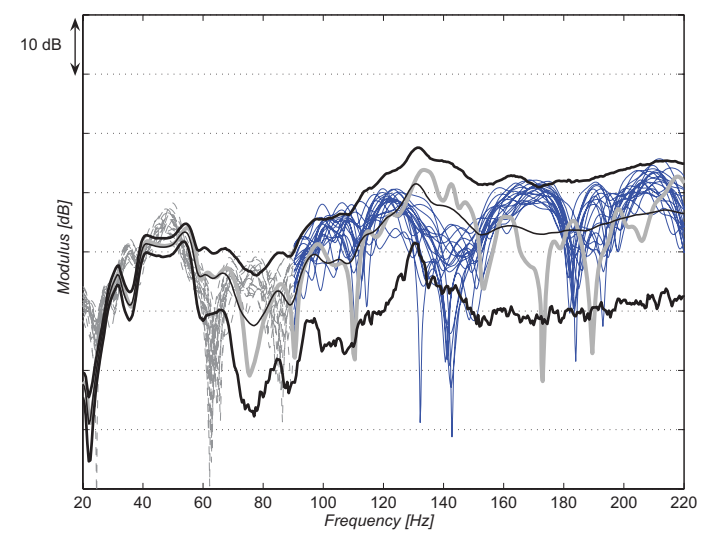

Fig. 16. For observation O6, comparisons of the experimental measurements (20 thin solid lines) with (1) the mean computational model (thick solid line), (2) the mean value of the optimal stochastic reduced computational model and (3) the confidence region of the optimal stochastic reduced computational model (upper and lower envelopes are the upper and lower thick solid lines

the computational results for observation $O 6$ (see Fig. 14). In Fig. 16, the 20 thin solid lines represent the experimental measurements, the upper and lower thick solid lines represent the upper and lower envelopes of the confidence region calculated for a probability level of 0.96 , the mid thin solid line represents the mean value of the random response of the stochastic reduced computational model, the mid thick grey solid line represents the response of the reduced mean computational model. Taking into account the complexity of the vibroacoustic model, the obtained results validate the stochastic computational model and demonstrate its capability to predict experimental measurements knowing that the dispersion parameters of model uncertainties have been identified using experimental measurements. 


\section{Conclusions}

In this paper we have presented methods to perform the identification and the validation of complex uncertain dynamical systems using experimental data in the area of structural vibrations and vibroacoustics, for which data and model uncertainties are taken into account by the nonparametric probabilistic approach. Such a probabilistic model of uncertainties allows model uncertainties and parameter uncertainties to be addressed by using only a small number of unknown identification parameters. Consequently, the optimization problem which has to be solved in order to identify the unknown identification parameters from experiments is feasible. Two formulations have been proposed. The first one is the mean-square method for which two cases of objective function has been proposed: a usual differentiable objective function and an unusual non-differentiable objective function which is more effective. The second one is the maximum likelihood method coupling with a statistical reduction which leads us to a considerable improvement of the method. Three applications have been presented in the area of structural vibrations and vibroacoustics for which experiments were available yielding experimental validations of the theory proposed.

\section{Acknowledgment}

This research has been supported by the French National Research Agency (ANR) in the context of the CORODYNA project, by PSA Peugeot-Citroen and by Université Paris-Est (Laboratoire de Mécanique (LAM) from Marne la Vallée and Laboratoire d'Analyse des Matériaux et Identification (LAMI) from Ecole des Ponts).

\section{References}

[1] C. Audet, J.E. Dennis Jr., Analysis of generalized pattern searches, SIAM Journal on Optimization 13(3) (2003) 889903.

[2] M. Arnst, Inversion of Probabilistic Models of Structures Using Measured Transfer Functions, Thesis of Ecole Centrale Paris, France, 2007.

[3] M. Arnst, D. Clouteau, H. Chebli, R. Othman, G. Degrande, A non-parametric probabilistic model for ground-borne vibrations in buildings, Probabilistic Engineering Mechanics 21(1) (2006) 18-34. 
[4] H. Berger, L. Barthe, R. Ohayon, Parametric updating of a finite element model from experimental modal characteristics, Mechanical System and Signal Processing 4(3)(1990)233-242.

[5] J. Bos, Numerical optimization of the thickness distribution of three-dimensional structures with respect to their structural acoustic properties, Structural Multidisciplinary Optimization 32(1)(2006) 12-30.

[6] E.Capiez-Lernout, C. Soize, Robust design optimization in computational mechanics, Journal of Applied Mechanics, (accepted for publication in june 2007).

[7] E. Capiez-Lernout, C. Soize, Robust updating of computational models with uncertainties for dynamical systems, Computational Methods in Structural Dynamics and Earthquake Engineering (COMPDYN 2007), Rethymno, Crete, Greece, June 13-15, 2007, Procceedings on CDROM edited by M. Papadrakakis, D.C. Charmpis, N.D. Lagaros, Y. Tsompanakis, National Technical University of Athens, Greece, 2007.

[8] H. Chebli, C. Soize, Experimental validation of a nonparametric probabilistic model of non homogeneous uncertainties for dynamical systems, Journal of the Acoustical Society of America 115(2) (2004) 697-705.

[9] C. Chen, Vibration and Vibroacoustics of Sandwich Composite Panels in Presence of Uncertainties - Experiments and Model Validation, Thesis of the University of Marne la Vallée (in french), Paris, France, 2006.

[10] C. Chen, D. Duhamel, C. Soize, Probabilistic approach for model and data uncertainties and its experimental identification in structural dynamics: case of composite sandwich panels, Journal of Sound and vibration 294 (2006) 64-81.

[11] T.F. Coleman, Y. Li, On the convergence of reflective newton methods for largescale nonlinear minimization subject to sounds, Mathematical Programming 67(2) (1994) 189-224.

[12] T.F. Coleman, Y. Li, An interior, trust region approach for nonlinear minimization subject to bounds, SIAM Journal on Optimization 6 (1996) 418445 .

[13] A.R. Conn, N.I.M. Gould, P.L. Toint, A globally convergent augmented Lagrangian algorithm for optimization with general constraints and simple bounds, SIAM Journal on Numerical Analysis 28(2) (1991) 545572.

[14] A.R. Conn, N.I.M. Gould, P.L. Toint, A globally convergent augmented Lagrangian barrier algorithm for optimization with general inequality constraints and simple bounds, Mathematics of Computation 66(217) (1997) 261288.

[15] W. D'Ambrogio, A. Fregolent, The use of antiresonances for robust model updating, Journal of Sound and Vibration 236(2)(2000) 227-243.

[16] I. Doltsinis, Z. Kang, Robust design of structures using optimization methods , Computer Methods in Applied Mechanics and Engineering 193(23-26) (2004) 2221-2237. 
[17] J. Dong, K.K. Choi, N.H. Kim, M.J.D. Powell, Design optimization for structural-acoustic problems using FEA-BEA with adjoint variable method, ASME Journal of Mechanical Design 126(3)(2004) 527-533.

[18] J. Duchereau and C. Soize, Transient dynamics in structures with nonhomogeneous uncertainties induced by complex joints, Mechanical Systems and Signal Processing 20 (2006) 854-867.

[19] J.-F. Durand, Modeling Automative Vehicles in Computational Vibroacoustics With Uncertainties and Experimental Validation, Thesis of the University of Marne la Vallée (in french), Paris, France, 2007.

[20] J.-F. Durand, L. Gagliardini, C. Soize, Random uncertainties modelling for vibroacoustic frequency response function of cars, in C. Soize and G.I. Schueller (Eds), Structural Dynamics EURODYN 2005, ISBN 905966033 1, Millpress, Rotterdam, Netherlands, pp. 1193-1198, 2005.

[21] C. Farhat, F.M. Hemez, Updating finite element dynamic models using an element-by-element sensitivity methodology, AIAA Journal 31(9)(1993) 17021711.

[22] C. Fernandez, C. Soize, L. Gagliardini, Modeling sound-insulation layers in vibroacoustic systems, 1st International Conference on Uncertainty in Structural Dynamics, The University of Sheffield, Sheffield, UK, June 11-13, 2007.

[23] D. Fritze, S. Marburg, H.-J. Hardtke, FEM-BEM-coupling and structuralacoustic sensitivity analysis for shell geometries, Computers \& Structures 83(23)(2005) 143-154.

[24] R. Ghanem, Ingredients for a general purpose stochastic finite elements formulation, Computer Methods in Applied Mechanics and Engineering 168 (1999) 19-34.

[25] R. Ghanem, P.D. Spanos, Stochastic Finite Elements: A spectral Approach (revised edition), Dover Publications, New York, 2003.

[26] P.E. Gill, W. Murray, M.H. Wright, Practical Optimization, Academic Press, London, 1981.

[27] D.E. Goldberg, Genetic Algorithms in Search, Optimization and Machine Learning, Addison-Wesley, 1989.

[28] S.P. Han, A globally convergent method for nonlinear programming, Journal of Optimization Theory and Applications 22(3) (1977) 297-309.

[29] F.M. Hemez, S.W. Doebling, Review and assessment of model updating for non linear transient dynamics, Mechanical System and Signal Processing 15(1)(2001) $45-74$.

[30] B. Jaishi, R. Wei-Xin, Damage detection by finite element model updating using modal flexibility residual, Journal of Sound and Vibration 290 (2006) 369-387. 
[31] E.T. Jaynes, Information theory and statistical mechanics, Physical Review 106 (4) (1957) 620-630 and 108 (2) (1957) 171-190.

[32] I.T. Jolliffe, Principal Component Analysis, Springer-Verlag, New York, 1986.

[33] D.H. Jung, B.C. Lee, Development of a simple and efficient method for robust design optimization, International Journal for Numerical Methods in Engineering 53(9) (2002) 2201-2215.

[34] K.S. Kwon, R.M. Lin, Robust finite element model updating using Taguchi method, Journal of Sound and Vibration 280(1-2) (2005) 77-99.

[35] P. Ladeveze, A. Chouaki, Application of a posteriori error estimation for structural model updating, Inverse Problem 15 (1999) 49-58.

[36] P. Ladeveze, M. Reynier, H. Berger, R. Ohayon, F. Quetin, L. Barthe, Updating methods of dynamic structured finite element model: dynamic reaction forces approach and error in constitutive equation approach, La Recherche Aérospatiale 5 (1991) 9-20.

[37] K.-H. Lee, G.-J. Park, Robust optimization considering tolerances of design variables, Computers \& Structures 79(1) (2001) 77-86.

[38] R.I. Levin, N.A.J. Lieven, Dynamic finite element model updating using neural networks, Journal of Sound and Vibration 210(5)(1998) 593-607.

[39] R.M. Lewis, V. Torczon, Pattern search algorithms for bound constrained minimization, SIAM Journal on Optimization 9(4) (1999) 1082-1099.

[40] S. Marburg, Developments in structural-acoustic optimization for passive noise control, Archives of Computational Methods in Engineering 9(4)(2002) 291-370.

[41] C. Mares, J.E. Mottershead, M.I. Friswell, Stochastic model updating: Part 1 - theory and simulated example, Mechanical System and Signal Processing 20 (2006) 1674-1695.

[42] C. Mares, J.E. Mottershead, M.I. Friswell, Stochastic model updating: Part 2 - application to a set of physical structures, Mechanical System and Signal Processing 20 (2006) 2171-2185.

[43] J.E. Mottershead, M.I. Friswell, Model updating in structural dynamics : a survey, Journal of Sound and Vibration 167 (1993) 347-375.

[44] R. Ohayon, C. Soize, Structural Acoustics and Vibration, Academic Press, San Diego, 1998.

[45] C. Papadimitriou, J.L. Beck, L.S. Katafygioti, Updating robust reliability using structural test data, Probabilistic Engineering Mechanics 16(2) (2001) 103-113.

[46] M. Papadrakakis, N.D. Lagaros, V. Plevris, Design optimization of steel structures considering uncertainties, Engineering Structures 27(9) (2005) 14081418. 
[47] A. Parkinson, C. Sorensen, N. Pouhassan, A general approach for robust optimal design, ASME Journal of Mechanical Design 115(1) (1993) 74-80.

[48] M.J.D. Powell, A fast algorithm for nonlinearly constrained optimization calculations, in proc.1977 Dundee Biennial Conference on Numerical Analysis, G.A.Watson, ed., Vol. 630, pp. 144-157, Springer-Verlag, Berlin, 1978.

[49] B. Ramakrishnan, S.S. Rao, A general loss function based optimization procedure for robust design, Engineering Optimization 25(4) (1996) 255-276.

[50] J.L. Raynaud, N. Bouhaddi, A. Perriot, Updating complex structures by a robust multilevel condensation approach, Journal of Sound and Vibration 270(12)(2004) 403-416.

[51] E. Sandgren, T.M. Cameron, Robust design optimization of structures through consideration of variation, Computers \& Structures 80(20-21) (2002) 1605-1613.

[52] G.I. Schueller (Ed.), A state-of-the-art report on computational stochastic mechanics, Probabilistic Engineering Mechanics 12(4) (1997) 197-313.

[53] G.I. Schueller, Computational stochastic mechanics - recent advances, Computers and Structures 79(22-25) (2001) 2225-2234.

[54] G.I. Schueller, On the treatment of uncertainties in structural mechanics and analysis, Computers and Structures 85(5) (2007) 235-243.

[55] R .J. Serfling, Approximation Theorems of Mathematical Statistics, John Wiley \& Sons, 1980.

[56] C.E. Shannon, A mathematical theory of communication, Bell System Technology Journal 27 (1948) 379-423 and 623-659.

[57] C. Soize, A nonparametric model of random uncertainties on reduced matrix model in structural dynamics, Probabilistic Engineering Mechanics 15(3) (2000) $277-294$.

[58] C. Soize, Maximum entropy approach for modeling random uncertainties in transient elastodynamics, Journal of the Acoustical Society of America 109(5) (2001) 1979-1996.

[59] C. Soize, Random matrix theory and non-parametric model of random uncertainties, Journal of Sound and Vibration 263 (2003) 893-916.

[60] C. Soize, Uncertain dynamical systems in the medium-frequency range, Journal of Engineering Mechanics 129(9) (2003) 1017-1027.

[61] C. Soize, A comprehensive overview of a non-parametric probabilistic approach of model uncertainties for predictive models in structural dynamics, Journal of Sound and Vibration 288(3) (2005) 623-652.

[62] C. Soize, Random matrix theory for modeling uncertainties in computational mechanics, Computer Methods in Applied Mechanics and Engineering 194(12-16) (2005) 1333-1366. 
[63] C. Soize, H. Chebli, Random uncertainties model in dynamic substructuring using a nonparametric probabilistic model, Journal of Engineering Mechanics 129(4) (2003) 449-457.

[64] J.C. Spall, Introduction to Stochastic Search and Optimization, John Wiley and Sons, Hoboken, New Jersey, 2003.

[65] G. Taguchi, E. Elsayed, T. Hsiang, Quality Engineering in Production Systems, McGraw-Hill, 1989.

[66] E. Walter, L. Pronzato, Identification of Parametric Models from Experimental Data, Springer, 1997.

[67] C. Zang, M.I. Friswell, J.E. Mottershead, A review of robust optimal design and its application in dynamics, Computers \& Structures 83(4-5) (2005) 315-326. 